\title{
Coherent structures in uniformly sheared turbulent flow
}

\author{
By CHRISTINA VANDERWEL \\ AND STAVROS TAVOULARIS \\ Department of Mechanical Engineering, University of Ottawa, Ottawa, Ontario, Canada
}

(Received November 2010 and in revised form May 2011)

Uniformly sheared turbulent flow has been generated in a water tunnel and its instantaneous structure has been examined using flow visualization and particle image velocimetry. The shear-rate parameter was approximately equal to 13 and the streamwise turbulence Reynolds number was approximately 150 . The flow was found to consist of regions with nearly uniform velocity, which were separated by regions of high shear containing large vortices. The concentration of vortices and the distributions of their directions of rotation, strengths, sizes and shapes have been determined. These results demonstrate that horseshoe/hairpin-shaped vortices were prevalent, even though wall effects were negligible in this flow. Both 'upright' and 'inverted' vortices have been observed, in contrast to turbulent boundary layers, in which only 'upright' vortices can be found, suggesting that the presence of the wall may suppress the development of 'inverted' structures. Our observations demonstrate that the dominant coherent structures of fully developed USF are very different from the structures observed in the flow exiting the shear-generating apparatus, which points to an insensitivity of the former to initial effects.

Key Words: Turbulence, Coherent Structures, Uniformly Sheared Flow, Homogeneous Shear Flow

\section{Introduction}

Coherent structures, commonly understood to be vortical entities of fluid with spatially correlated properties and recurring patterns, are essential elements of all turbulent flows. Although usually occupying a small part of the bulk of the fluid, coherent structures contribute significantly, and sometimes overwhelmingly, to turbulent transport and mixing, forces on immersed objects, and noise. They have been studied intensively in both free and bounded shear flows, including mixing layers, jets, wakes, boundary layers, and channel flows. Of particular interest have been the coherent structures in constantpressure, incompressible, turbulent boundary layers (TBL) over smooth walls (e.g. Kline et al. 1967; Falco 1977; Head \& Bandyopadhyay 1981; Robinson 1991; Smith et al. 1991; Adrian, Meinhart \& Tomkins 2000; Adrian 2007; Wu \& Moin 2009). Horseshoe/hairpinshaped vortices† are known to be the dominant structures in this type of flow, in which they are largely responsible for the production of Reynolds stresses and turbulence kinetic energy. The generation of coherent structures in boundary layers has been linked intimately to the proximity of a rigid wall, which is the source of mean shear as well

$\dagger$ The descriptive names horseshoe and hairpin refer to the same type of coherent structures; horseshoe-shaped vortices appear at relatively low Reynolds numbers and become more elongated resembling hairpins as the Reynolds number increases (Head \& Bandyopadhyay 1981). 
as an impenetrable barrier. Mean shear, irrespectively of its origin, is conventionally recognized as a main source of turbulence. Thus, it seems worthwhile to examine the characteristics of coherent structures in a flow that on the average resembles a boundary layer but is free of other complications introduced by a wall. A configuration that meets ideally these requirements is the homogeneous shear flow (HSF), which is unbounded and attainable only theoretically, and its experimentally realizable approximation, the uniformly sheared flow (USF). Both HSF and USF are unidirectional on the mean and have a uniform mean velocity gradient transverse to the flow direction. In HSF, turbulence is homogeneous and evolves in time, whereas in USF it is nearly homogeneous on a transverse plane but evolves streamwise. The Reynolds stress anisotropy of USF resembles that in the outer regions of turbulent boundary layers (TBL), but analytically and experimentally, USF is easier than TBL to characterize.

Experimental investigations of USF have been conducted in wind and water tunnels, in which the mean shear was generated by various devices inserted at the test section entrance and the turbulence was let to develop downstream (e.g. Tavoularis \& Corrsin 1981; Rohr et al. 1988; De Souza, Nguyen \& Tavoularis 1995; Ferchichi \& Tavoularis 2000; Shen \& Warhaft 2000; Isaza, Warhaft \& Collins 2009). These studies reported measurements of the Reynolds stresses and other statistical properties of turbulence and discussed the evolution of the turbulence kinetic energy, the turbulence anisotropy, and the fine structure under the influence of uniform shear. All available measurements were made with hot-wire/hot-film anemometers or laser Doppler velocimeters, which, although having sufficient spatial and temporal resolutions to measure accurately the local velocity, cannot provide spatial maps of the instantaneous velocity field. Consequently, none of these earlier studies attempted a systematic experimental documentation of coherent structures in USF. The only available experimental investigation of the large-scale structure of USF is the flow visualization work by Kislich-Lemyre (2002), which identified the presence of horseshoe-shaped vortices and mushroom-like flow patterns far away from solid walls.

In parallel with the experimental work on USF, a number of numerical simulations of HSF have been published during the past three decades (e.g. Rogers \& Moin 1987; Adrian \& Moin 1988; Lee, Kim \& Moin 1990; Kida \& Tanaka 1994; Isaza \& Collins 2009), following the development of a suitable direct numerical simulation (DNS) algorithm by Rogallo (1981). The DNS results complemented the experimental ones and established the close correspondence between the turbulence structure and evolution rate in HSF and those in USF. Besides Reynolds-averaged statistics, DNS have also determined the temporal evolutions of the entire velocity and vorticity fields, albeit under the limitation of a relatively small Reynolds number and short development times. Lee et al. (1990) and Kida \& Tanaka (1994) investigated the instantaneous structure of HSF and demonstrated that it is organized into coherent turbulent structures. The results of Rogers \& Moin (1987) clearly identified the presence of hairpin vortices, which formed by the rollup of vortex sheets with spanwise vorticity. The strongest vorticity vectors were oriented initially at $45^{\circ}$ with respect to the direction of flow and tended to decrease their inclination with evolution time. The heads of hairpin vortices were equally as likely to be towards the higher or the lower velocities, in contrast to TBL observations, in which they always appear towards the higher velocity region away from the wall. To demonstrate the analogy between hairpins in HSF and those in TBL, Adrian \& Moin (1988) associated upward ejection events with 'upright' hairpins and downward sweeps with 'inverted' hairpins.

The objective of the present work is to document experimentally the properties of large-scale coherent structures in uniformly sheared flow. In this article, we report quali- 


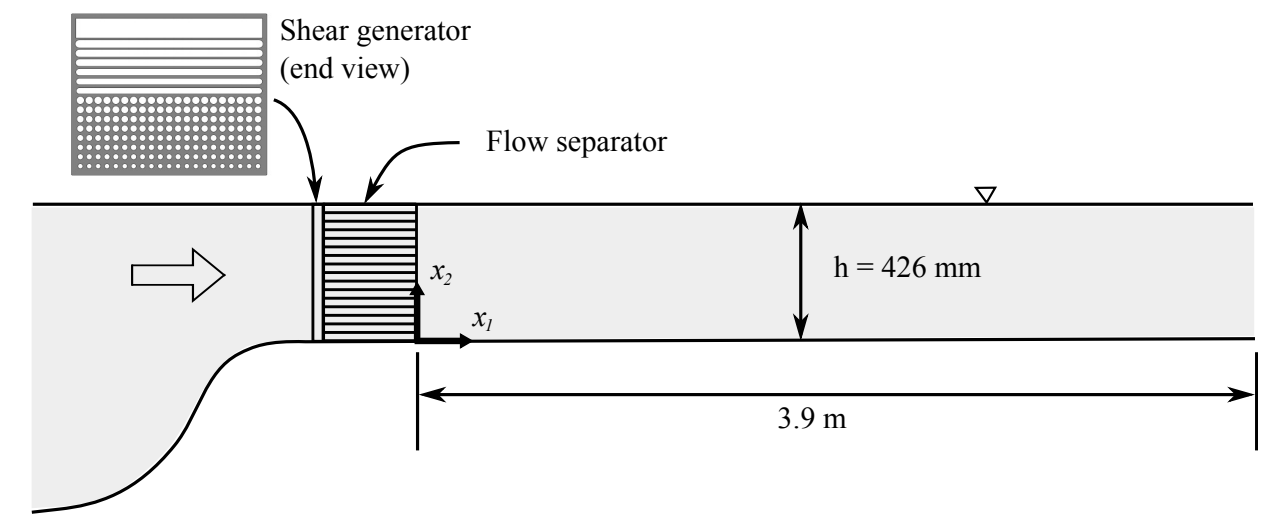

FIGURE 1. Schematic of the test section from a side view and the shear generator/separator apparatus.

tative and quantitative experimental results obtained in USF generated by conventional means in a water tunnel. The flow facility and apparatus are discussed in section 2 . Section 3 presents measurements of the time-averaged local velocity, turbulent stresses, length scales, and time scales, obtained using mainly laser Doppler velocimetry (LDV). Section 4 shows representative flow visualisation images of hydrogen bubbles and fluorescent dyes recorded by using both still and continuously traversed cameras. Instantaneous velocity maps obtained with the use of particle image velocimetry (PIV) are presented in section 5. Finally, the experimental results are analyzed and discussed in section 6 , which also reflects on the implications of the presence of horseshoe-shaped vortices. The main conclusions are summarised in section 7 .

\section{Apparatus, instrumentation and experimental procedures}

The present experiments were conducted in a recirculating water channel, having a horizontal test section with a width of $540 \mathrm{~mm}$, a length of $3.9 \mathrm{~m}$, not including the shear generating apparatus, and filled with water up to a height of $h=426 \mathrm{~mm}$. The test section allows optical access through three glass walls, and the free surface. An automated secondary circulation loop was used overnight to chlorinate the water and to filter suspended particles larger than about $30 \mu \mathrm{m}$. Uniform shear was generated at the test section entrance by a perforated plate made of aluminium and having an approximately linear variation of solidity from top to bottom. This shear generator was followed by a flow separator, which consisted of a frame housing sixteen panes of tempered glass, $1.6 \mathrm{~mm}$ thick and $25.4 \mathrm{~mm}$ apart. The function of the flow separator was to straighten the stream, which would otherwise have curved streamlines near the shear generator, as well as to establish a uniform initial integral length scale of turbulence. A sketch of the test section is shown in figure 1. A Cartesian coordinate system was defined such that the $x_{1}$ axis corresponds to the direction of mean flow, the $x_{2}$ axis is in the vertical direction, which coincides with the direction of the velocity gradient, and the spanwise $x_{3}$ axis is orthogonal to both $x_{1}$ and $x_{2}$; the origin of the coordinate system is at the exit of the flow separator, in the mid-point of the bottom wall of the channel. The facility was equipped with three rail-mounted traversing systems, which could be traversed along the top, the bottom and one side of the test section.

A two-component laser Doppler velocimeter (LDV) with a burst analyzer processor was 


$\begin{array}{llll}x_{1} / h & 4.2 & 5.9 & 7.5 \\ U_{c}\left(\mathrm{~ms}^{-1}\right) & 0.1671 & 0.1682 & 0.1683 \\ \mathrm{~d} \bar{U}_{1} / \mathrm{d} x_{2}\left(\mathrm{~s}^{-1}\right) & 0.5529 & 0.5501 & 0.5651 \\ \tau & 6.0 & 8.3 & 10.6\end{array}$

TABLE 1. Mean flow parameters measured using LDV at three downstream positions.

used for measuring the streamwise $U_{1}$, vertical $U_{2}$, and spanwise $U_{3}$ velocity components, mainly for the purpose of determining time-averaged flow properties. A two-dimensional planar particle image velocimetry (PIV) system was used for measuring instantaneous velocity maps. PIV measurements were taken in several different planar orientations in order to capture different features of the flow. Maps of the velocity and the swirling strength $\lambda$ were used to identify vortices in the flow. Following the method suggested by Gao, Ortiz-Duenas \& Longmire (2007), it was assumed that vortices were present at locations at which the swirling strength had local peaks. Only peaks with $\lambda>0.05 \lambda_{\max }$ were considered, where $\lambda_{\max }$ was the maximum swirling strength in the flow map. The presence of vortices at these locations was validated visually by verifying that the velocity vectors (relative to the vortex convection speed) within their proximity indicated a swirling motion, as suggested by Robinson (1991). Flow visualisation methods using hydrogen bubbles and fluorescent dyes were also employed to investigate the flow. Details of the instrumentation and measurement procedures are provided in appendix A.

\section{Time-averaged flow measurements}

Time-averaged statistical properties were measured using primarily LDV, complemented in some instances by PIV, in order to document the overall characteristics of the present flow and to establish that its average structure was comparable to those in previous USF studies. This section will only summarize representative results, as timeaveraged properties of USF have been reported in great detail in previous articles (e.g. Tavoularis \& Corrsin 1981; Tavoularis \& Karnik 1989).

Vertical profiles of the mean velocity at several downstream positions in the centreplane of the channel, were very nearly linear, such that the differences between the measured values and the fitted lines were less than $1 \%$ in the core of the flow $(0.3<$ $\left.x_{2} / h<0.8\right)$. Representative values of the mean velocity on the centreline $U_{c}$ and the mean shear $\mathrm{d} \bar{U}_{1} / \mathrm{d} x_{2}$ are shown in table 1 . The slight increase in centreline velocity downstream (by about $0.5 \%$ over the entire test section) may be attributed to boundary layer growth. The mean shear in the core of the flow also appeared to increase slightly. The same table also shows the corresponding values of the dimensionless turbulence development time $\tau$, also representing the total strain experienced by the turbulence at each downstream location; to account for the slight variations in centreline mean velocity and mean shear along the test section, $\tau$ was defined as

$$
\tau=\int_{0}^{x_{1}} \frac{1}{U_{c}} \frac{\mathrm{d} \bar{U}_{1}}{\mathrm{~d} x_{2}} \mathrm{~d} x_{1} .
$$

As indicators of the degree of transverse homogeneity of the turbulence, representative vertical profiles of the standard deviations $u_{1}^{\prime}$ and $u_{2}^{\prime}$ of, respectively, the streamwise and transverse velocity components and the turbulent shear stress correlation coefficient $\rho=-\overline{u_{1} u_{2}} / u_{1}^{\prime}{ }_{1} u_{2}$ have been plotted in figures 2 and 3 . In the downstream half of the test section $(\tau>5.0)$, deviations from vertical homogeneity were relatively small. The 


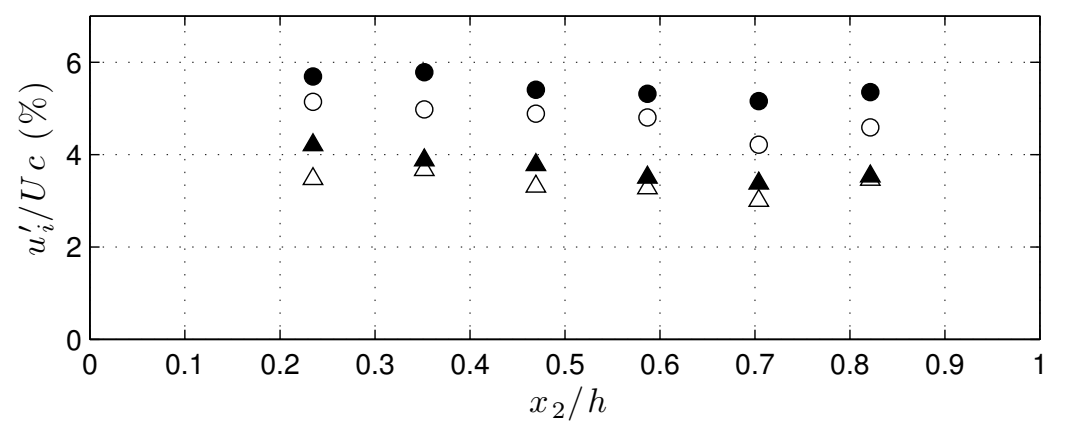

Figure 2. Profiles of the normalised second moments of the turbulent velocity fluctuations; ○: $i=1, \tau=8.3 ; \bullet: i=1, \tau=10.6 ; \Delta: i=2, \tau=8.3 ; \boldsymbol{\Delta}: i=2, \tau=10.6$.

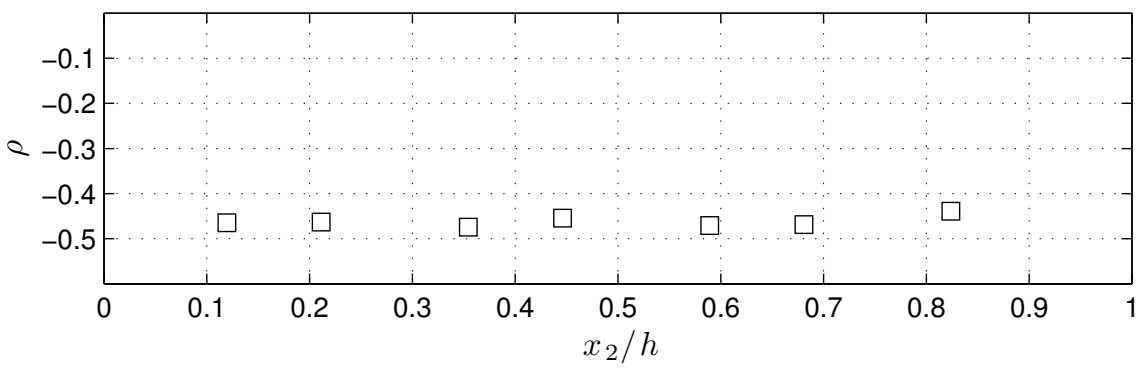

FiguRE 3. Profile of the turbulent shear stress correlation coefficient $\rho=-\overline{u_{1} u_{2}} / u_{1}^{\prime} u_{2}^{\prime}$ at $\tau=8.3$.

normal stress profiles at $\tau=8.3$ and 10.6 demonstrate a streamwise growth in turbulence, which extended nearly uniformly across the core of the test section. The shear stress correlation coefficient was consistently around -0.45 for all depths except near the freesurface where it was slightly lower. The weak inhomogeneity of the turbulence properties, likely connected to a corresponding non-uniformity of turbulence production, may be attributed partly to imperfections in the adjustment of the shear generator and partly to boundary-layer and free-surface effects. The spanwise inhomogeneity of the turbulence in the core of the flow was found to be much weaker than the vertical one. Unless otherwise specified, results reported in the subsequent sections were collected in the centre-plane of the tunnel near the mid-height of the test section, where inhomogeneity was weakest.

Turbulence measurements along the test section centreline have been plotted in figure 4 . These measurements show that the turbulent stresses initially decreased in magnitude, as turbulence generated by the shear generator and flow separator decayed, however, further downstream, in the range $5<\tau<10$, all stresses increased at approximately exponential rates, in accordance with previous findings (Tavoularis 1985; Pope 2000). Also in agreement with previous findings, the magnitudes of the turbulent stresses were ordered as $\overline{u_{1}^{2}}>\overline{u_{3}^{2}}>\overline{u_{2}^{2}}>-\overline{u_{1} u_{2}}$. The turbulent kinetic energy per unit mass, defined as

$$
k=\frac{1}{2}\left(\overline{u_{1}^{2}}+\overline{u_{2}^{2}}+\overline{u_{3}^{2}}\right),
$$




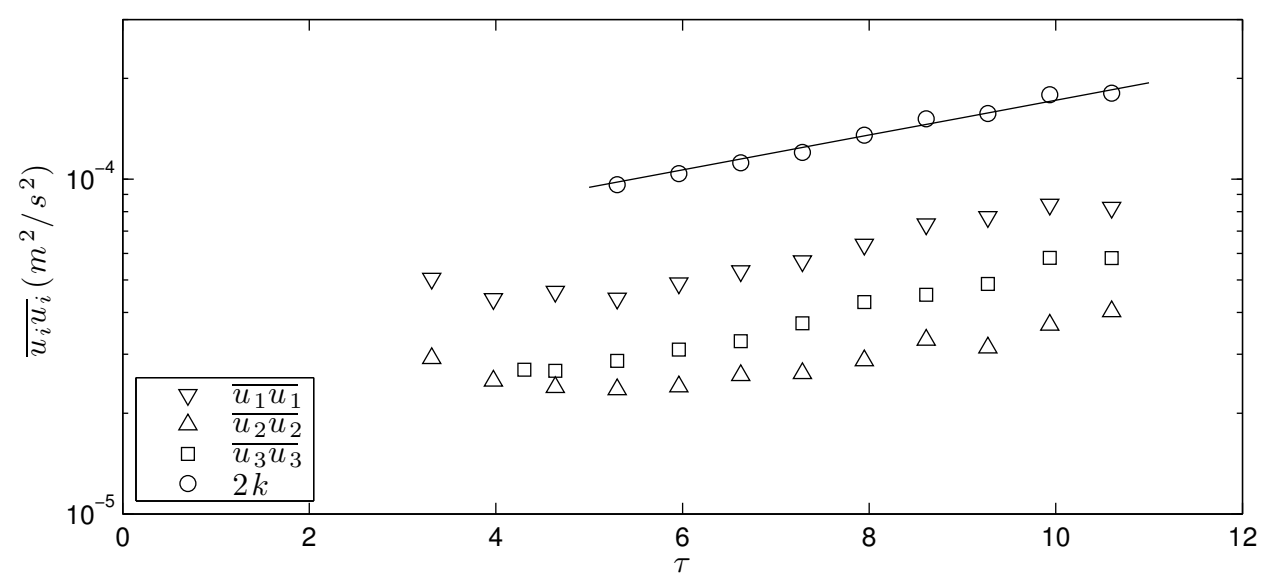

FiguRE 4. Streamwise growth of the second moments of the turbulent velocity fluctuations and the turbulent kinetic energy at $x_{2} / h=0.47$.

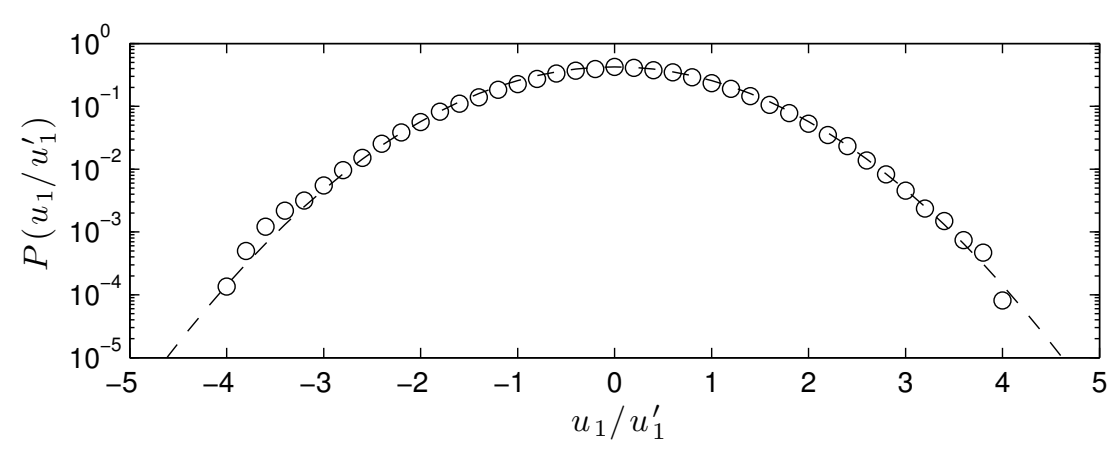

FiguRE 5. The p.d.f. of the streamwise velocity fluctuations compared to a Gaussian p.d.f. (dashed line).

grew downstream according to the relationship

$$
k=k_{0} e^{a \tau},
$$

where $a=0.12$ and $k_{0}=2.44 \times 10^{-5} \mathrm{~m}^{2} / \mathrm{s}^{2}$.

The probability density function (p.d.f.) of the streamwise velocity fluctuations, presented in figure 5, was nearly Gaussian with a skewness of -0.05 and a flatness of 3.12 , in agreement with the findings by Tavoularis \& Corrsin (1981) and Ferchichi \& Tavoularis (2000).

A summary of the main turbulence characteristics have been listed in table 2 . The Reynolds stress anisotropy tensor is defined as

$$
m_{i j}=\frac{\overline{u_{i} u_{j}}}{2 k}-\frac{1}{3} \delta_{i j}
$$

where $\delta_{i j}$ is Kronecker's delta. The kinetic energy dissipation rate per unit mass $\epsilon$ was estimated from the simplified turbulent kinetic energy equation, as the difference between the rate of change of turbulent kinetic energy and the rate of production, namely as

$$
\epsilon=-\overline{u_{1} u_{2}} \frac{d \bar{U}_{1}}{d x_{2}}-U_{c} \frac{d k}{d x_{1}} .
$$




\begin{tabular}{ll|ll}
$a$ & 0.12 & $k\left(\mathrm{~mm}^{2} \mathrm{~s}^{-2}\right)$ & 86 \\
$\epsilon / P r$ & 0.6 & $\epsilon\left(\mathrm{mm}^{2} \mathrm{~s}^{-3}\right)$ & 7.6 \\
$m_{11}$ & 0.16 & $L_{e}(\mathrm{~m})$ & 3.8 \\
$m_{22}$ & -0.12 & $L_{11,1}(\mathrm{~mm})$ & 35 \\
$m_{33}$ & -0.02 & $\lambda_{11}(\mathrm{~mm})$ & 17 \\
$m_{12}$ & -0.15 & $\eta(\mathrm{mm})$ & 0.6 \\
$S^{*}$ & 13 & $R_{\lambda_{11}}$ & 150
\end{tabular}

TABLE 2. Measured or estimated turbulence parameters at $\tau=10$.

From the values of $k$ and $\epsilon$, we calculated the eddy lifetime $2 k / \epsilon$, which is a measure of the time between the generation and destruction of the dominant turbulent eddies. Moreover, we calculated a representative streamwise distance over which a typical eddy would be convected during its lifetime as

$$
L_{e}=U_{c} \frac{2 k}{\epsilon} .
$$

The shear-rate parameter was determined as

$$
S^{*}=\frac{2 k}{\epsilon} \frac{\mathrm{d} \bar{U}_{1}}{\mathrm{~d} x_{2}}
$$

which is the ratio of the turbulence timescale to the timescale of the mean shear and represents the relative strength of the turbulence with respect to the production by mean shear.

The streamwise Taylor microscale $\lambda_{11}$ was estimated as (De Souza et al. 1995)

$$
\lambda_{11} \simeq \sqrt{\frac{12 \nu(2 k)}{\epsilon}}
$$

and the Kolmogorov microscale $\eta$ was computed as

$$
\eta=\left(\frac{\nu^{3}}{\epsilon}\right)^{1 / 4} \text {. }
$$

Finally, the turbulence Reynolds number was calculated as

$$
R_{\lambda_{11}}=\frac{u_{1}^{\prime} \lambda_{11}}{\nu} .
$$

The autocorrelation function of the streamwise velocity is presented in figure 6 . The LDV output, which recorded the velocities of seeded particles crossing the measurement volume at random times, was re-sampled at a fixed time interval of $2 \mathrm{~ms}$ using a sampleand-hold method (Tavoularis 2005), so that it was converted to a time series with a uniform spacing of the samples. Subsequently, this time series was low-pass filtered using a cut-off of $20 \mathrm{~Hz}$ to eliminate the effects of ambiguity noise and other high-frequency effects which contaminated the initial part of the autocorrelation function. The presented autocorrelation curve was determined by ensemble averaging 20 independent records, each $60 \mathrm{~s}$ long. The streamwise integral time scale $T_{11}$ was computed by integrating the autocorrelation function up to its first zero. Then, the integral length scale $L_{11,1}$ was computed using Taylor's frozen flow approximation as $L_{11,1}=\bar{U}_{1} T_{11}$. In the downstream half of the test section $(5<\tau<10), L_{11,1}$ grew from approximately 25 to $40 \mathrm{~mm}$ at an approximately linear rate. 


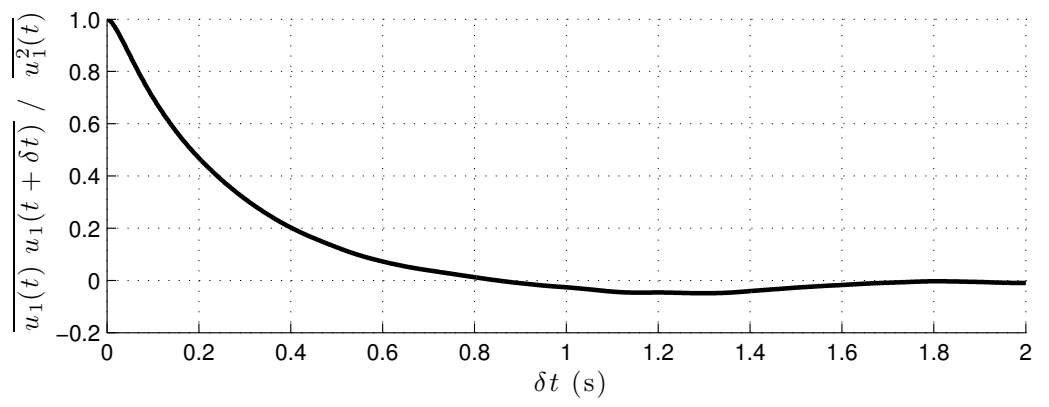

FiguRE 6. Typical autocorrelation function of the streamwise velocity signal measured at the centre of the cross section at $\tau=8.3 . L_{11,1} \approx 32 \mathrm{~mm}$ at this location.
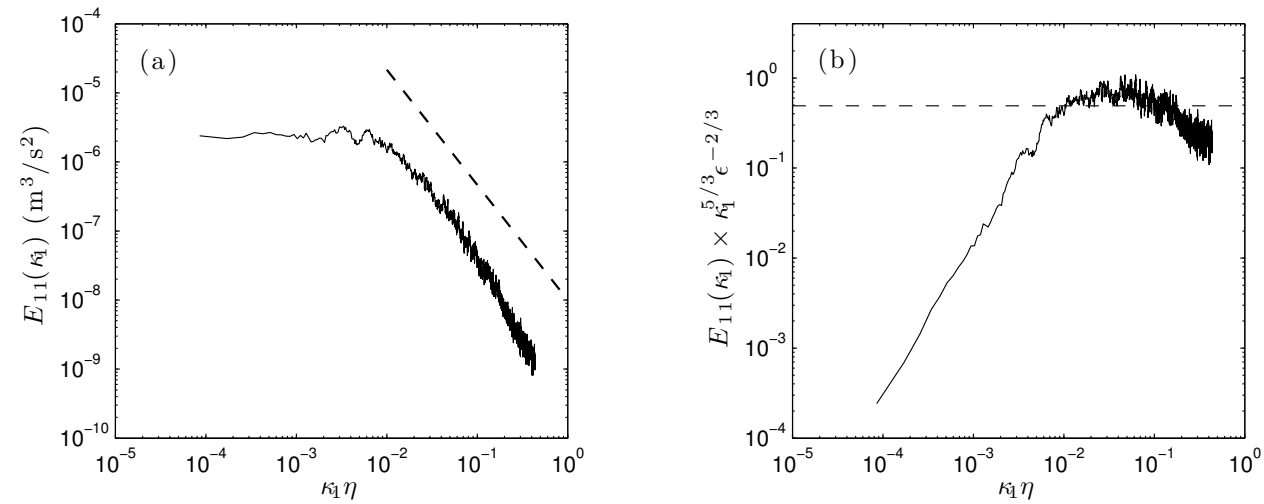

Figure 7. (a) Typical one-dimensional spectrum of the streamwise velocity measured at the centre of the water tunnel cross section at $\tau=10.6$; the dashed line represents Kolmogorov's $-5 / 3$ law of the inertial subrange. (b) The same spectrum multiplied by $\kappa_{1}^{5 / 3}$ and normalized by $\epsilon^{2 / 3}$; the dashed line indicates a value of $C_{1}=0.49$ for the universal Kolmogorov constant (Pope 2000).

A typical wavenumber spectrum $E_{11}\left(\kappa_{1}\right)$ of the streamwise velocity is shown in figure $7 \mathrm{a}$, where $\kappa_{1}=2 \pi f / \bar{U}_{1}$ is the streamwise wavenumber. The same spectrum is shown in figure $7 \mathrm{~b}$ multiplied by $\kappa_{1}^{5 / 3}$ and normalized by $\epsilon^{2 / 3}$. Only a very narrow inertial range, if any at all, may be seen in the spectrum, as anticipated in view of the moderate value of $R_{\lambda_{11}}$. An alternative estimate of the integral lengthscale from the spectrum (Pope 2000) was $L_{11,1} \simeq \pi E_{11}(0) / 2 \overline{u_{1}^{2}}=35 \mathrm{~mm}$, which was close to the value estimated from the autocorrelation.

The results presented in table 2 indicate that the anisotropy, shear-rate parameter and turbulence Reynolds number of the turbulence in the present USF had comparable values to those in earlier, mildly sheared USF, particularly the one by Tavoularis \& Corrsin (1981). In terms of length scales, it may be seen that the typical convection length of energy containing eddies during their lifetime exceeded the test section length, and so it may be expected that eddies generated by the mean shear in the test section would remain distinct throughout the measurement range. In conformity with previous findings, the integral length scale was comparable to the flow separator spacing and the three characteristic turbulence scales were ordered according to the usual order $\eta<\lambda_{11}<$ 
$L_{11,1}$. The relative closeness between $\lambda_{11}$ and $L_{11,1}$ is consistent with the moderate value of $R_{\lambda_{11}}$, which was, however, sufficiently large for the turbulence to be fully developed and markedly larger than corresponding values in most previous DNS of HSF.

\section{Flow visualisation results}

\subsection{Hydrogen bubble visualisation}

Hydrogen bubble visualisation was performed with the cathode wire placed horizontally, parallel to the spanwise axis $x_{3}$, and just below mid-depth. Such tests were performed at two water channel speeds: at the same speed as the LDV tests (i.e., with $U_{c}=0.16 \mathrm{~m} / \mathrm{s}$; to be referred to in this section as the 'high-speed' case) and at half that speed ( $U_{c}=$ $0.08 \mathrm{~m} / \mathrm{s}$; to be referred to as the 'low-speed' case). In the high-speed case, turbulent mixing was quite strong and, although coherent structures were identifiable close to the wire, they ceased to be discernible beyond about $250 \mathrm{~mm}$ downstream. In contrast, in the low-speed case, the bubble timelines remained connected and allowed the vortices to be seen over longer distances.

Figure 8 shows two examples of low-speed bubble visualisation images with the wire located at $x_{1} / h \simeq 4.7(\tau \simeq 5.4)$. These images contain clear evidence of the presence of horseshoe-shaped vortices. The legs of these structures become identifiable by the quasi-streamwise alignment of bubble lines across several timelines; the heads of the structures are also clearly visible in the bubble patterns in the form of arches connecting two elongated legs. As an aid to the eye, we have used white lines to trace a few of these structures on the two images and presented the results in the same figure, underneath the original untouched images. One may thus distinguish three isolated horseshoe vortices in figure $8 \mathrm{a}$, as well as some longer vortices, which contain both forward-facing and backward-facing heads, in figure 8b. The orientations of these structures with respect to the vertical direction are difficult to discern from such images, but it appeared that they were typically inclined by roughly $45^{\circ}$ with respect to the vertical direction. As the structures travelled downstream, they continued stretching along the $+45^{\circ}$ inclined plane until the bubble lines became very thin and they could no longer be identified. The length of the structures varied from 40 to $80 \mathrm{~mm}$, and the spacing of the legs was on average about $30 \mathrm{~mm}$.

Similar structures were also identified in the high-speed flow, as illustrated by an example in figure 9. Although the structures were much more difficult to identify than in the low-speed case, observation of video sequences demonstrated that the horseshoe structures in the high-speed flow were on average both narrower and shorter than in the low-speed flow, as well as appearing more frequently and being closer to neighbouring structures. Leg spacing in the high-speed case varied typically between 5 and $20 \mathrm{~mm}$, though in a few cases it reached values up to $50 \mathrm{~mm}$. The structures often spanned several timelines, having typical lengths in the range of 20 to $30 \mathrm{~mm}$, although they occasionally reached lengths of up to $60 \mathrm{~mm}$. Although each structure had typically one head and two legs, some structures appeared to have only one leg or more than one head. As in the low-speed case, some heads were observed to face upstream and others downstream. A typical value of about $\Delta \tau=0.5$ was estimated to be the average dimensionless time over which the bubble patterns remained coherent, however, as this value is also influenced by the turbulent mixing and the dispersion of the bubbles, this is expected to be a low estimate of the lifetime of the vortices. 

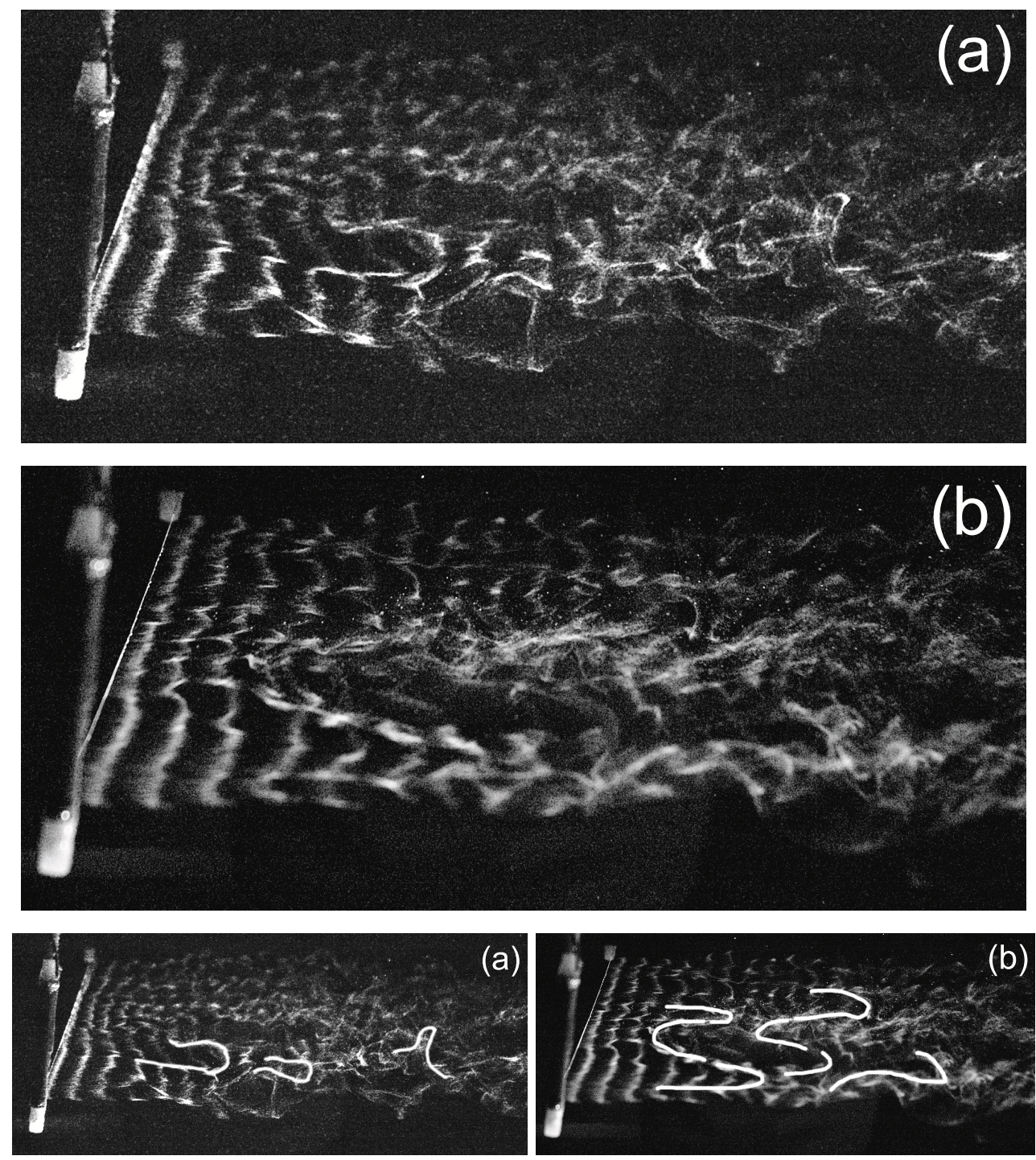

FIgURE 8. Representative images of hydrogen bubble timelines in USF with $U_{c}=0.08 \mathrm{~m} / \mathrm{s}$; two original images are shown at the top and middle, whereas the bottom row shows the same images on which the outlines of a few vortices have been traced by hand.

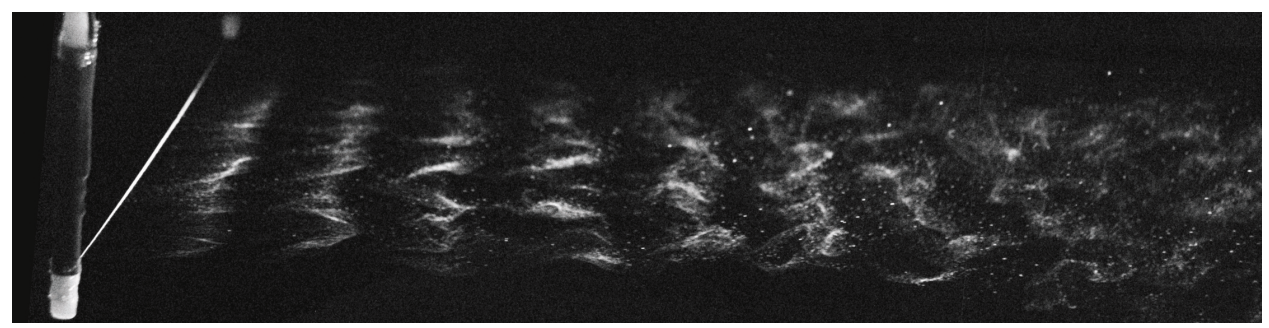

FIGURE 9. Representative image of hydrogen bubble timelines in USF with $U_{c}=0.16 \mathrm{~m} / \mathrm{s}$. 


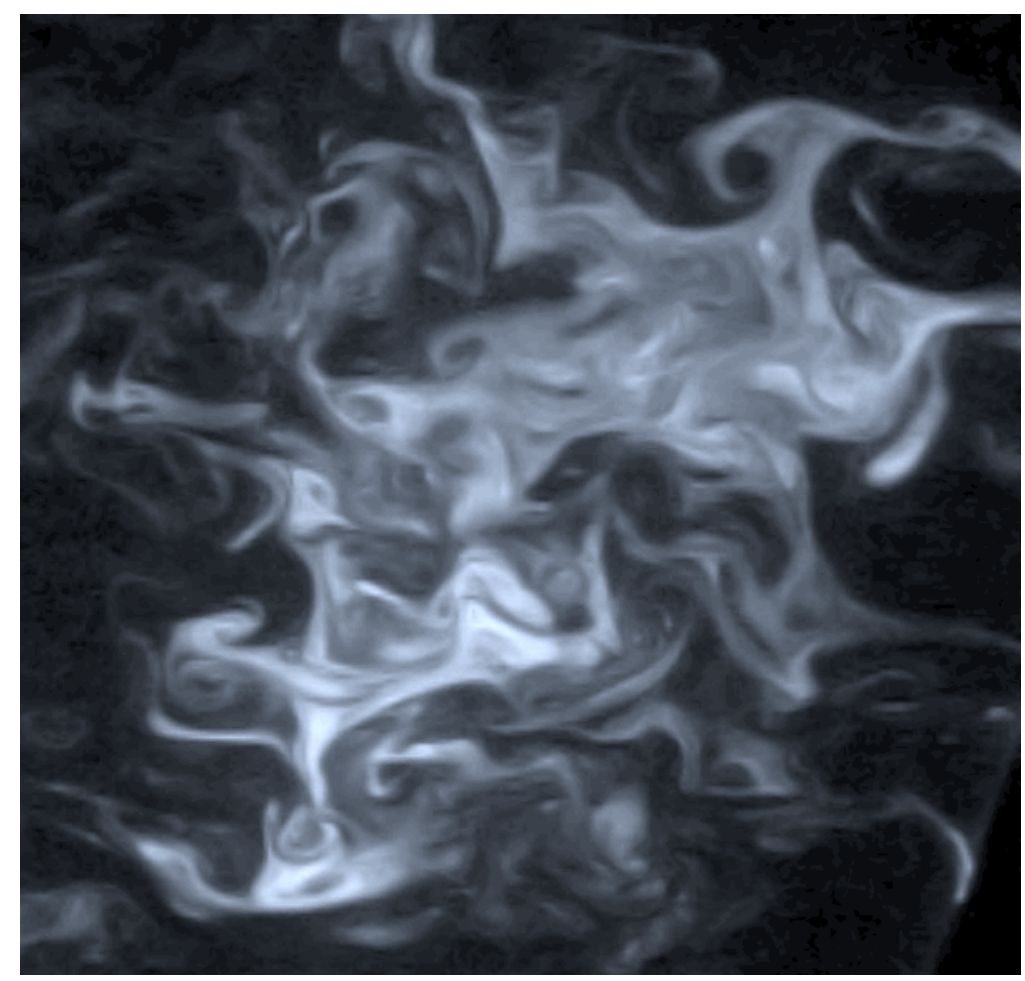

Figure 10. Representative image of the flow in a plane inclined by $45^{\circ}$ with respect to the streamwise direction showing several mushroom-shaped dye patterns.

\subsection{Dye visualisation}

Essentially instantaneous, three-dimensional dye patterns formed in the USF were captured by scanning a laser sheet along the test section and recording the illuminated flow with a high speed camera mounted on the same carriage as the light-sheet lens. Dye patterns in the range $2.7<\tau<5.7$ were scanned in less than one second. Because the eddy lifetime (previously calculated to be approximately $27 \mathrm{~s}$ ) was much larger than the scan time, the vortices were essentially 'frozen' during the scan. For the same reason, however, vortices could not be identified by appearing as spiral motions, as would have been the case had the flow patterns been followed by a camera moving with the average flow speed. Instead, vortices were identified as dye patterns that had circular or spiral outlines. These were common throughout the flow but were most easily distinguished near the edges of the dye cloud and near the bottom of the image where the laser light was most intense.

Counter-rotating vortex pairs were found to be very common, distinguished by dye patterns that resembled mushrooms. The two vortices appeared as circular patterns forming the cap of the mushroom, whereas the dye drawn between the vortices appeared as the mushroom stem. Several examples of these patterns can be identified in figure 10. Mushroom-shaped patterns were observed facing in all directions. The distance between the centres of the two vortices was typically in the range from 20 to $50 \mathrm{~mm}$. Most mushroom-shaped dye patterns could only be tracked for a streamwise length of about $50 \mathrm{~mm}$, as in subsequent frames these patterns became faded or mixed with other patterns.

Several cross-sections of a representative mushroom-shaped vortex pair are presented 


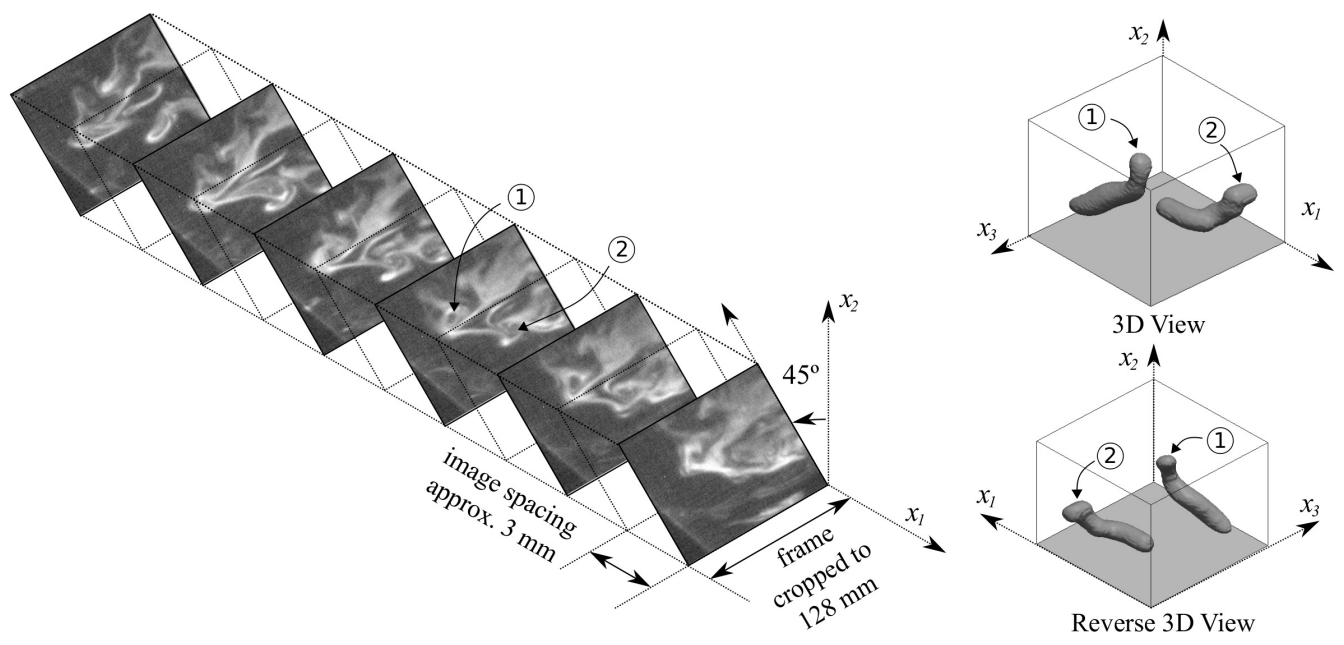

FiguRE 11. (Left) Frame sequence of the scan through a pair of counter-rotating vortices, marked by swirling dye patterns. The flow was scanned in an angled plane moving approximately $1.5 \mathrm{~m} / \mathrm{s}$ upstream. Images were recorded at 500 frames/second; every second image is displayed with a streamwise spacing not to scale. (Right) Two views of the three-dimensional (3D) reconstruction of the two vortices.

in the frame sequence shown in figure 11. The orientation of the stem of this particular mushroom-shaped dye pattern indicated that the vortices induced a downwards velocity in the space between them. A three-dimensional reconstruction of the vortex pair corresponding to this frame sequence is also presented in figure 11. The identifiable length of this vortex pair was about $66 \mathrm{~mm}$, however, the actual length was likely to be greater, as the ends of the vortices were not traceable in the dye images. The two counter-rotating vortices were separated by a distance of about $50 \mathrm{~mm}$. The vortices were both inclined upwards by about $35^{\circ}$ with respect to the $x_{1}$-direction in the $x_{1}-x_{2}$ plane, and sideways by about $25^{\circ}$ with respect to the $x_{1}$-direction in the $x_{1}-x_{3}$ plane; this corresponds to an inclination of $56^{\circ}$ with respect to the $x_{3}$-direction in the $x_{2}-x_{3}$ plane.

The two counter-rotating vortices were interpreted as the legs of a horseshoe-shaped vortex, similar in shape to those observed in low Reynolds number TBL. Unfortunately, the head of the horseshoe vortex was difficult to discern from the dye pattern. In the smoke visualisations of Head \& Bandyopadhyay (1981), the heads of hairpins were only clearly distinguished using a laser sheet inclined at $-45^{\circ}$, in which the dye patterns formed loops. Their images using a laser sheet inclined at $+45^{\circ}$, such as in the current study, revealed upright mushroom-shaped dye patterns, similar to those observed currently. In contrast to the results of Head \& Bandyopadhyay (1981), mushroom patterns were observed in all orientations, the implications of which will be considered in $\S 6$.

\section{PIV measurements}

All reported PIV measurements were taken at the nominal speed of $U_{c}=0.16 \mathrm{~m} / \mathrm{s}$. The results are presented as planar velocity contour maps, with all velocity magnitudes normalized by $U_{c}$. Identified vortices have been superimposed on these maps as ellipses, each of which encloses the area of the vortex as determined by the swirling strength map. The ellipse is coloured black if the vortex was rotating clockwise and white if rotating counter-clockwise. 


\begin{tabular}{lccccc}
\hline & \multicolumn{2}{c}{$\begin{array}{c}\text { Vertical } \\
\text { plane }\end{array}$} & \multicolumn{2}{c}{ Horizontal } \\
plane & $\begin{array}{c}\text { Inclined } \\
\text { plane }\end{array}$ \\
Development time $\tau$ & 0.3 & 1.1 & 5.2 & 5.2 & 6.1 \\
Average number of vortices in a & 84 & 49 & 35 & 39 & 15.5 \\
$\quad 100$ mm $\times 100$ mm viewing area & 54 & 63 & 76 & 50 & 56 \\
Percentage of clockwise vortices & 178 & 98 & 55 & 159 & 165 \\
Mean vortex strength $\left(\mathrm{mm}^{2} / \mathrm{s}\right)$ & 1242 & 715 & 416 & 1029 & 1184 \\
Maximum vortex strength $\left(\mathrm{mm}^{2} / \mathrm{s}\right)$ & 5.8 & 5.9 & 4.9 & 11.7 & 12 \\
Mean vortex diameter $\left(\mathrm{mm}^{2}\right)$ & 21.2 & 21.1 & 20.7 & 45 & 38
\end{tabular}

TABLE 3. Properties of vortices in the three PIV measurement planes.

The average vortex concentrations and the mean and maximum absolute vortex strengths and diameters for each of the measurement configurations are summarised in table 3. Statistical properties of the vortices were computed using at least 70 independent PIV vector maps, each of which extended over a planar area of $100 \mathrm{~mm} \times 100 \mathrm{~mm}$ centred at the indicated downstream position.

\subsection{Vertical plane measurements}

These measurements were taken on the vertical centre-plane of the channel, namely on a plane parallel to the streamwise axis $x_{1}$ as well as the direction of the velocity gradient $x_{2}$. Figure 12 shows velocity contours at three streamwise locations: close to the exit of the flow separator $(0.13 \leqslant \tau \leqslant 0.40)$, where inlet effects are expected to dominate on the turbulence; a little further downstream $(0.95 \leqslant \tau \leqslant 1.22)$, where inlet effects are expected to have diminished somewhat and production by the mean shear to have started having some measurable effect; and at about the mid-section of the channel $(4.92 \leqslant \tau \leqslant 5.19)$, where the turbulence structure is expected to be dominated, at least on the average, by production by the constant mean shear. Instantaneous velocity profiles are presented alongside the velocity maps, as well as the average velocity profile away from the flow separator. The locations of the instantaneous profiles shown were selected so that no vortices would be intersected by the corresponding axes to avoid local distortion of the velocity profile by vortex-induced velocities. Furthermore, the elevations of the plates in the flow separator are indicated by dotted lines for reference.

In the instantaneous velocity profile near the exit of the flow separator (figure 12, top; $\tau \approx 0.27$ ), one may clearly distinguish the high-speed zones of fluid exiting each channel of the flow separator from the low-speed wakes of the flow separator plates. At this location, the instantaneous local vorticity, and even the time-averaged local vorticity, are far from being uniform; nevertheless, the time-averaged vorticity would be nearly uniform when spatially averaged over the entire height of the test section. A large number of vortices have been identified in the flow near the exit of the shear generating apparatus; they are almost entirely organized into alternating vortex streets, and the numbers of clockwise and counter-clockwise vortices are roughly equal. The spatial distribution of these vortices is consistent with the jet-wake character of the velocity profile. The mean diameter of the vortices was $5.8 \mathrm{~mm}$, which corresponds to roughly one quarter of the spacing between the plates in the flow separator.

At the downstream position with $\tau \approx 1.08$ (figure 12 , middle), the vertical velocity profile was considerably smoother and the concentration of vortices was significantly 

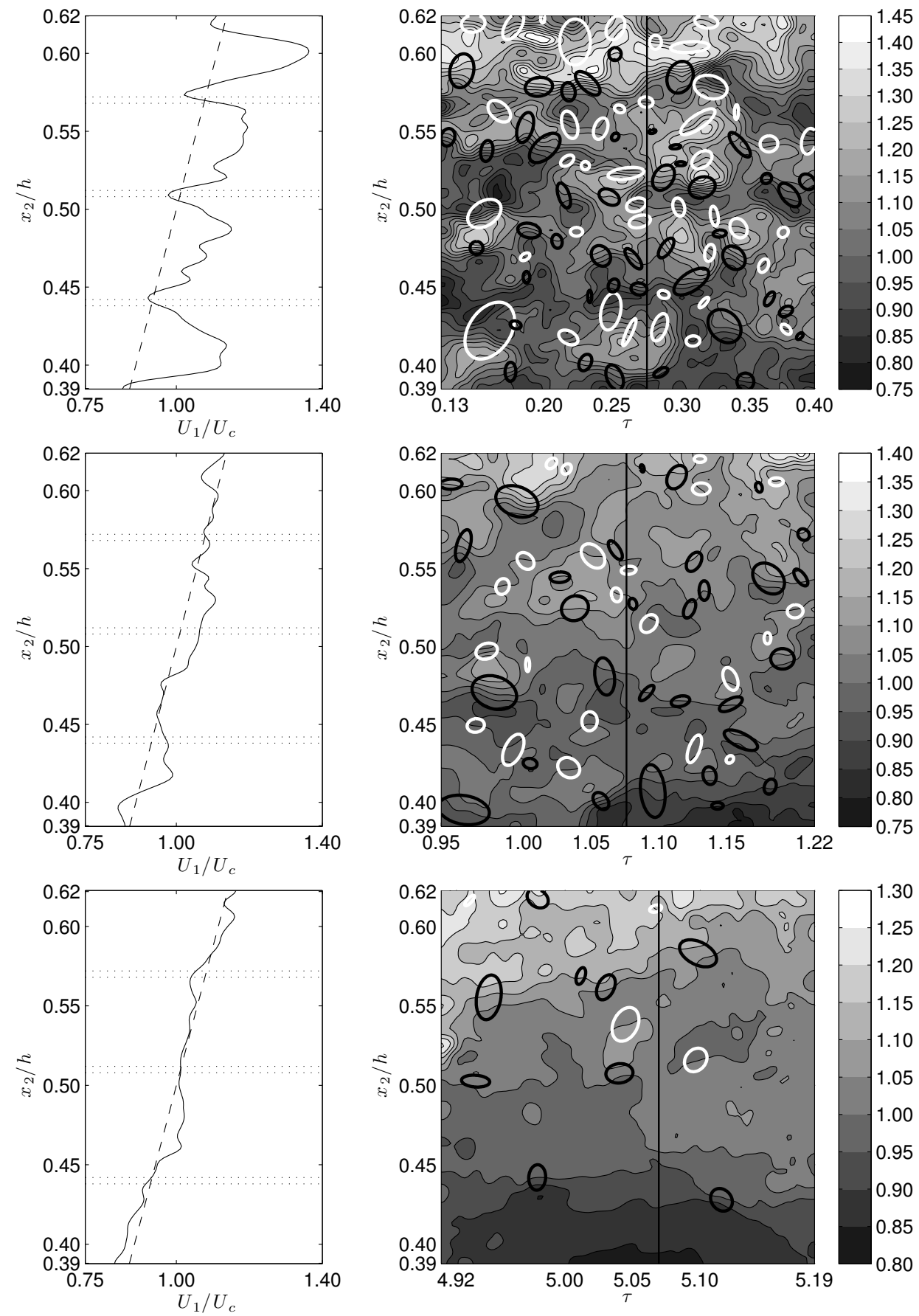

FigURE 12. Instantaneous velocity maps in the vertical centre-plane at three downstream locations. The velocity magnitudes have been normalized by the time-averaged centreline velocity $U_{c}$. Superimposed ellipses indicate the positions, sizes, and rotation senses of identified vortices; black ellipses represent clockwise rotations and white ellipses represent counterclockwise rotations. On the left of each map, the instantaneous streamwise velocity profile corresponding to the position in the map is drawn as a solid line and the time-averaged velocity profile is drawn as a dashed line. Dotted lines outline the elevations of the plates of the flow separator. 
lower (nearly half) than at $\tau \approx 0.27$. At $\tau \approx 1.08$, the vortices had approximately the same mean diameter as at $\tau \approx 0.27$, but their mean strength was reduced significantly. The numbers of clockwise and counter-clockwise vortices were no longer equal, with the counter-clockwise vortices being significantly fewer. The streamwise decrease in the level of velocity fluctuations in the flow close to the shear generator/flow separator is evident in the velocity contours at these two locations.

At a downstream distance of $\tau \approx 5.07$ (figure 12, bottom), the velocity profile was much smoother. Although the time-averaged velocity profile was linear at this downstream position, the instantaneous velocity profile had a stepwise character. The instantaneous velocity field tended to form zones of roughly uniform velocity, separated by thin shear layers with large velocity gradients. At the instant shown in the figure, a large region of nearly uniform velocity was present in the centre of the image, flanked by two shear layers with greater than average shear located at depths of $x_{2} / h=0.45$ and 0.57 . These features persisted in the streamwise direction, although, as indicated by the velocity contours, the shear layers were slightly inclined to the flow direction as they tended to meander up and down. At other instances, the zones of roughly uniform velocity appeared at different vertical positions, such that the shear layers appeared as ramps separating these zones. The zones of uniform velocity typically spanned $50 \mathrm{~mm}$ in the $x_{2}$-direction and had a streamwise length that was longer than the field of view, which was about $100 \mathrm{~mm}$. The locations of the shear layers were not correlated with the depths of the plates in the flow separator. Large clockwise vortices were found to be common along these shear layers and were often seen to travel in groups of three to six, forming chains that stretched up to $100 \mathrm{~mm}$ in length.

In addition to PIV measurements with the light sheet and the camera fixed at some streamwise position, additional measurements were made with the laser mounted on a carriage located underneath the channel and the camera mounted on another carriage that was located on the side of the channel, while both carriages were traversed along the test section at a speed equal to $U_{c}$. The resulting velocity maps were consistent with those in the fixed frames. The fluctuations in the velocity profile became reduced with increasing downstream distance, in conformity with the fixed-frame observations. The properties of the vortices in the moving frame also developed in conformity with those in the fixed frames. A large number of vortices were observed near the exit of the flow separator. As the flow moved downstream, the smaller vortices gradually disappeared; it is unclear whether these relatively small vortices decayed due to viscous dissipation or merged with others to form larger vortices. At the same time, the larger vortices became more organized, typically aligning with a strong shear layer. The larger vortices were sometimes observed to merge with other vortices or to break down. The merging of vortices generated larger, stronger ones, and the splitting of vortices resulted in fragments, which quickly disappeared. For the most part, the larger vortices typically endured for the entire length of the recording. Due to limitations in the apparatus, each video recording could only extend over a distance of $\Delta \tau<2$. Therefore, the flow in the entire length of the test section could not be traversed in a single run, and so it could not be determined whether the vortices far downstream were traceable back to the flow separator.

\subsection{Horizontal plane measurements}

A representative set of velocity contours on the horizontal plane at an elevation of $x_{2} / h=0.56$ is presented in figure 13. The velocity field in the horizontal plane was characterised by elongated low- and high-speed regions, that were roughly aligned with the streamwise direction. At the instant presented in figure 13, two high-speed regions located roughly at $x_{3} / w=-0.15$ and -0.05 , and two low-speed regions located roughly 

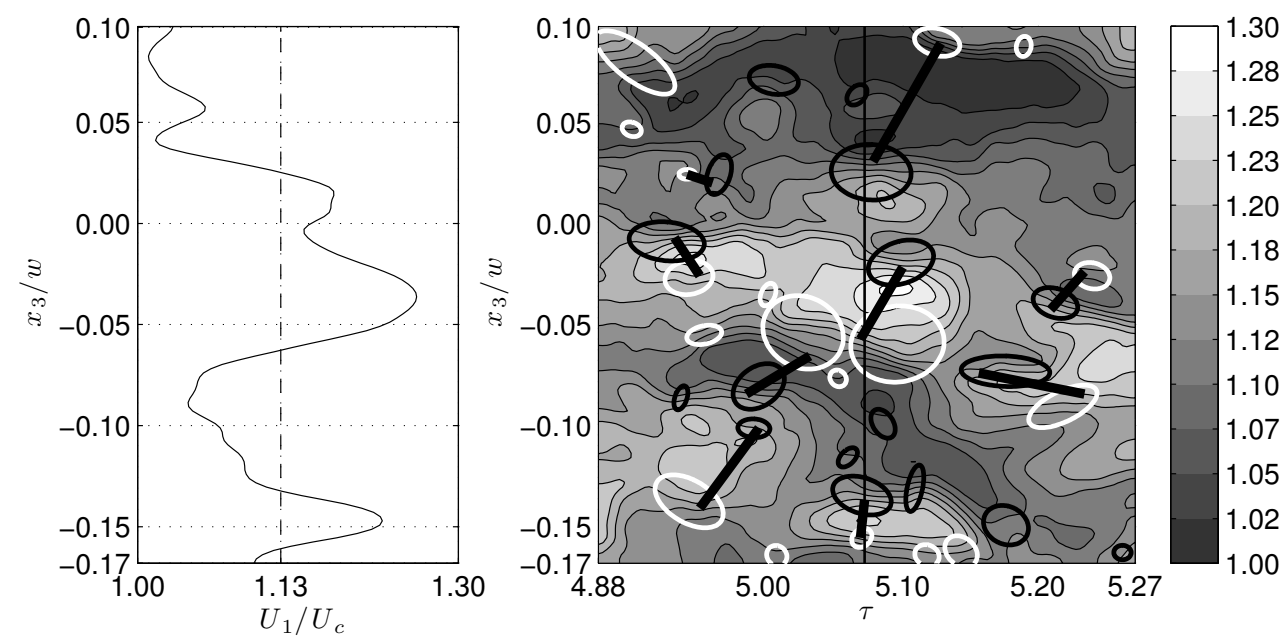

FigURE 13. Instantaneous velocity map in the horizontal plane at $x_{2} / h=0.56$ and near $\tau=5.1$. The velocity magnitudes have been normalized by the time-averaged centreline velocity $U_{c}$. Superimposed ellipses indicate the positions, sizes, and rotation senses of identified vortices; black ellipses represent clockwise rotations and white ellipses represent counterclockwise rotations. Solid lines connect counter-rotating vortex pairs, identified by a pair-matching algorithm described in appendix B. The instantaneous streamwise velocity profile from the centre of the plot has been drawn on the left, in comparison to the mean streamwise velocity at the depth of $x_{2} / h=0.56$ denoted by a dashed line.

at $x_{3} / w=-0.09$ and 0.08 can be identified. At other instances, however, the low- and high-speed regions were located at other spanwise positions. These zones were typically $100 \mathrm{~mm}$ long and had a spanwise spacing of about $25 \mathrm{~mm}$, resembling the streaks identified by Lee et al. (1990). Relatively large spanwise gradients of the streamwise velocity were observed to occur near the edges of these zones, along which several vortices were located.

Although the concentration of vortices in the horizontal plane was comparable to that in the vertical plane at the same $\tau$, the strengths and sizes of the vortices differed. There were roughly an equal number of clockwise and counter-clockwise rotating vortices in the horizontal plane, whereas in the vertical plane the clockwise vortices predominated away from the shear generator. The average diameter of the vortices in the horizontal plane was more than twice the one in the vertical plane. Most of the vortices travelled at the average convection velocity, often lining up in the streamwise direction between low- and high-speed streaks.

Observations of the orientations of the vortex tubes passing through the horizontal plane are discussed in detail in appendix B. From the shapes of the cross-sections of the vortices, we found that vortex tubes were most often inclined by about $35^{\circ}$ in the streamwise direction. Furthermore, the application of a pair-matching algorithm confirmed that vortices often travelled in counter-rotating pairs and that these pairs, which are presumably the legs of horse-shoe vortices, were twice as likely to travel with their plane facing the flow direction rather than sideways.

\subsection{Inclined plane measurements}

PIV measurements were taken in a plane inclined by $45^{\circ}$ with respect to the flow direction, with its upper part facing upstream, as in the scanning dye visualisation. The mean velocity in the inclined plane is towards the bottom of the channel, being the projection 
of the mean streamwise velocity, and also has a mean gradient towards the top, which is the projection of the mean velocity gradient in the $x_{2}$-direction. Some images were taken with simultaneous dye injection in order to allow comparison with results of previous dye visualisations. To achieve this, water containing both dibromofluorescein and silicon carbide micro-spheres was injected upstream of the shear generator. Dye patterns were recorded in the same images used for PIV measurements, and did not have a significant effect on the quality of the PIV results.

Figure 14 shows three aspects of the same representative PIV frame in the inclined plane. The photograph shows a large, upright, mushroom-shaped dye pattern; as the velocity map illustrates, the two spirals of the mushroom coincide with a pair of strong, counter-rotating vortices; finally, the vector map shows that fluid from the bottom of the image moves upwards between the two vortices of the pair, transporting with it dye and creating a local low velocity region.

Although the mean velocity changed linearly from top to bottom of the field of view, the instantaneous velocity contours exhibited large spanwise fluctuations. The velocity contours contained saw-tooth patterns in the spanwise direction. In the instant shown in the figure, the velocity contours outline a large zone of low-speed fluid located at approximately $x_{2} / h=0.36$ and $x_{3} / w=0.06$. Another such zone of high-speed fluid is located at approximately $x_{2} / h=0.44$ and $x_{3} / w=-0.06$. The zones within each saw-tooth were about 30-40 mm wide in the $x_{3}$-direction and 40-50 $\mathrm{mm}$ tall in the $x_{2^{-}}$ direction and tended to wander both vertically and horizontally. It is clear that these zones are related to the actions of vortices, because the velocity vectors show that the large-counter rotating pair of vortices influences the low-speed region below it.

As expected, there is an approximate balance of clockwise and counter-clockwise vortices. The vortex properties were similar to those in the horizontal plane, however, the concentration of vortices that were identified in the inclined plane was about half that in the horizontal plane. This may be partially attributed to the strong out-of-plane velocity component, which increased the difficulty in following a vortex in adjacent time frames and may have caused the smaller vortices to be missed. For the flow velocity of approximately $0.16 \mathrm{~m} / \mathrm{s}$ and the recording rate of approximately $7 \mathrm{~Hz}$, the flow moved out of the plane from one frame to the next by a distance comparable to the integral length scale $L_{11,1}$. Another consequence of the strong out-of-plane motion was a larger uncertainty of the measurement of the absolute flow velocity using the PIV system, which introduced large scatter in the statistics of the inclinations and ellipticities of the vortices.

\section{Discussion}

\subsection{Evidence of horseshoe/hairpin vortices in USF}

Although mainly used for qualitative purposes in the present study, the images of hydrogen bubble timelines released from a horizontal cathode wire at relatively low flow speeds clearly outlined complete horseshoe vortices. Because the lengths of these vortices far exceeded the distance between timelines, the legs of each vortex consisted of segments of several consecutive timelines. Nevertheless, the heads were clearly defined by bubbles within a single, arched timeline and there was no doubt that a head and two legs formed a single entity. Within their range of application, hydrogen bubbles are ideally suited for the visualisation of vortices, as they tend to concentrate along the vortex axis because of their low density and so they are able to define relatively long vortex segments, straight or contorted. On the other hand, as the turbulence increases with increasing flow speed, the bubble timelines get dispersed and vortex identification becomes more difficult. For this 

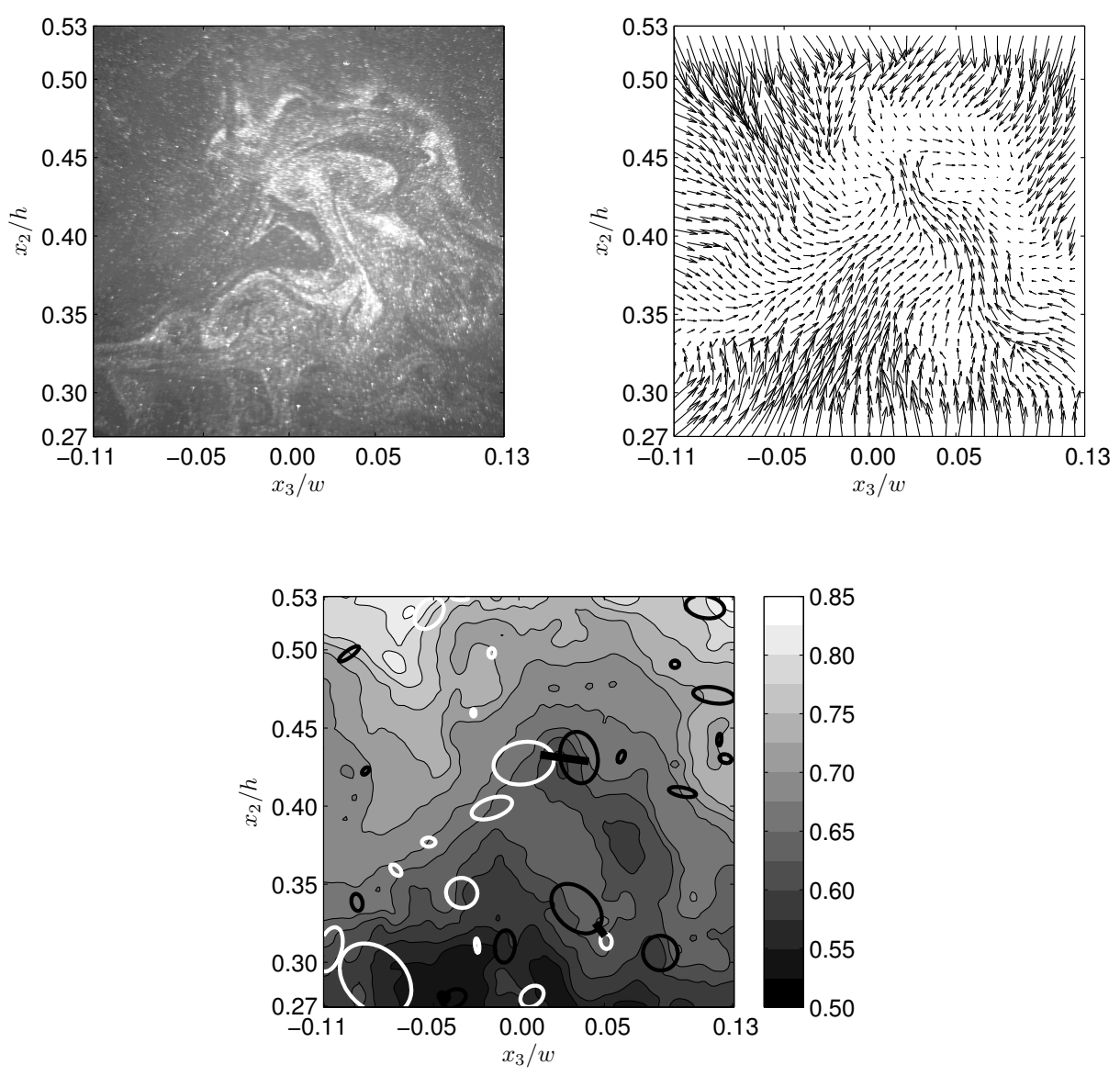

Figure 14. Simultaneous dye visualisation (top left) and PIV measurement in an inclined plane; vectors of the in-plane motion (upper right) are relative to the mean velocity in the image and the magnitudes of the velocity contours (bottom) have been normalized by the time-averaged centreline velocity $U_{c}$. Superimposed ellipses indicate the positions, sizes, and rotation of vortices; black ellipses represent clockwise rotations and white ellipses represent counterclockwise rotations. A solid line connects counter-rotating vortex pairs.

reason, our main bubble study examined a USF with half the speed of most other tests. Moreover, the bubble timelines only provided a very limited out-of-plane view of the flow structure and could not serve for the quantification of most vortex characteristics.

The scanning dye visualisation complemented the other tests by providing an approximately instantaneous, volumetric view of the flow. In this case, vortex legs were identified as regions containing dye spirals, which appeared in pairs and together formed mushroom-shaped patterns; simultaneous flow visualization and PIV proved conclusively that the two spirals of a mushroom coincided with counter-rotating vortices. Conversion of sequences of adjacent planar images to a three-dimensional view resulted in the reconstruction of pairs of elongated, inclined, nearly parallel vortex tubes. Unfortunately, vortex heads were not possible to identify from 'frozen' dye patterns illuminated transversely. Even so, in view of previous work in TBL (e.g. Head \& Bandyopadhyay 1981), one may be confident that mushroom-shaped patterns were evidence of horseshoe vortices. The dye visualisation demonstrated that such vortices occurred commonly in the flow. 

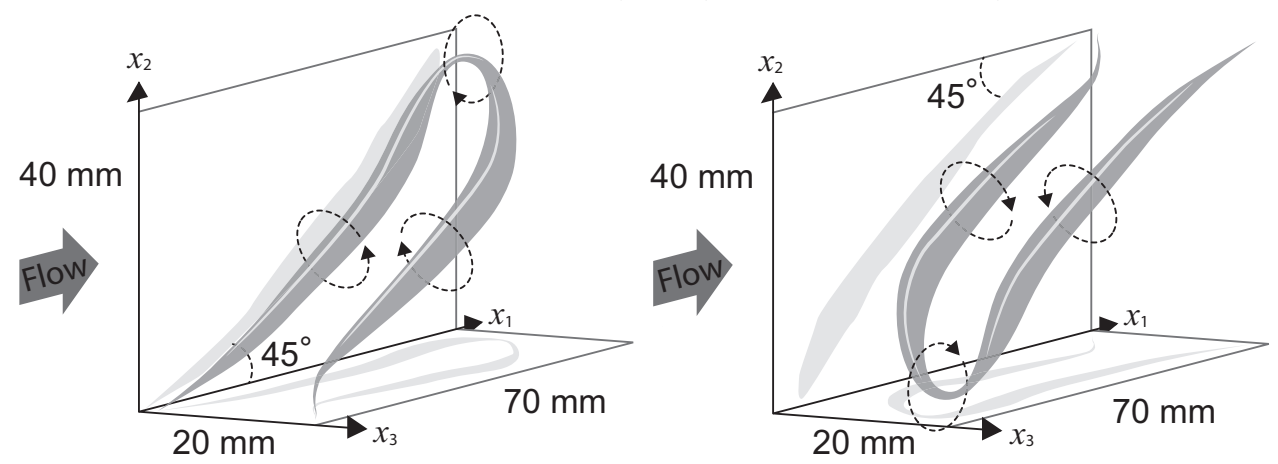

FIGURE 15. Sketches of an upright (left) and an inverted (right) horseshoe vortex, which are representative of many structures observed in the present USF.

Planar velocity maps in three planes in the flow, measured with PIV, also support the hypothesis that horseshoe vortices occur in abundance in USF. These maps contained a profusion of vortex cross-sections, which, in conformity with similar measurements in TBL, were interpreted to correspond to either heads or legs of horseshoe vortices. In a vertical plane parallel to the flow, large vortices were observed to congregate along shear layers that separated zones of relatively uniform velocity. In TBL studies (e.g. Adrian et al. 2000; Adrian 2007; Gao et al. 2007), such vortices were interpreted as the heads of horseshoe/hairpin vortices. Like TBL, the present USF also contained smaller vortices scattered over the measurement area, which did not seem to be the heads of horseshoe/hairpin vortices. PIV measurements in the horizontal and the inclined planes captured the cross-sections of the legs of horseshoe vortices. This was best illustrated by the simultaneous PIV and dye visualisation presented in figure 14, which showed a mushroom-shaped dye pattern coincident with two counter-rotating vortices. Although the length of the vortex pair could not be measured with the stationary PIV because of its insufficient temporal resolution, both the dye patterns and the velocity maps indicated that the vortex pair was sufficiently long to induce a significant draft of fluid in-between the two vortices.

In conclusion, all evidence collected in the present study strongly supports the hypothesis that the horseshoe vortex is the predominant large-scale coherent structure of USF. This hypothesis is also supported by previous DNS work (Rogers \& Moin 1987).

Following the previous discussion, it is now possible to describe the three-dimensional shape and size of a typical horseshoe vortex in USF (refer to figure 15). From the average length of the structures observed by the scanning dye visualisation, the streamwise length of the legs was estimated to be approximately $50 \mathrm{~mm}$. Based on observations of both the hydrogen bubbles and the scanning dye visualisation, the legs were found to be typically inclined upwards with respect to the direction of the flow, by approximately $30^{\circ}-45^{\circ}$. This corresponds to a vertical height of about $50 \mathrm{~mm}$, which compares with the average separation distance between shear layers, measured with PIV in the vertical plane. The legs are separated by about $25 \mathrm{~mm}$, as estimated by the dye visualisation, bubble patterns, and PIV measurements in the horizontal and inclined planes.

These horseshoe vortices were observed to twist and turn in the flow, appearing at a large range of inclinations. In addition to the upright configuration, which is typical of the hairpin structures of TBL (see Smith et al. 1991; Adrian et al. 2000), they also often appeared inverted (shown on the right of figure 15). 


\subsection{Inverted versus upright structures}

Results from all present experiments in USF support the hypothesis of the prevalence of horseshoe-shaped vortices, which resemble closely the coherent structures in TBL. The main difference between the coherent structures of USF and TBL is the existence of equally common inverted and upright horseshoe-shaped vortices in USF, whereas only upright structures have been observed in TBL. Sketches of typical upright and inverted structures in USF, based on all available evidence, are presented in figure 15. In the following, we shall reconsider such evidence.

(i) Although hydrogen bubbles could not identify the directions of rotation of the vortices, they sufficiently outlined both forward-facing, upright structures and backwardfacing, inverted structures.

(ii) Observation of mushroom-shaped dye patterns, which were encountered frequently in dye visualisation images and which have been attributed to the action of vortex pairs, identified the locations and directions of rotation of the legs of horseshoe vortices. Discrimination between upright and inverted horseshoes was based on the orientation of each mushroom pattern: mushrooms with caps facing upwards (i.e., in the direction of the mean velocity gradient) indicated that dye had flowed upwards between the vortex pair, thus pointing to the action of an upright horseshoe structure; inversely, mushrooms with caps facing downwards were evidence of the action of an inverted horseshoe structure. From the three-dimensional reconstructions of the pairs of vortex tubes, the inverted structures were typically found to be inclined at the same angle as the upright structures. Unfortunately, the dye visualisations were unable to resolve whether the two legs of a pair were attached by a vortex head and the location and direction of rotation of such head, if it existed.

(iii) The PIV measurements led to essentially the same conclusions as the dye visualisations. In the horizontal plane, vortex pairs were identified with a pair-matching algorithm and the relative positions of both vortices were investigated. It was concluded that there was a wide distribution of the inclinations of the vortex pairs (see figure 21), with slightly higher populations of upright and inverted structures, for which the line connecting the two vortices is normal to the streamwise direction $0^{\circ}$, compared to other inclinations.

The sketches of horseshoe vortices presented in figure 15 are consistent with all these observations. The upright horseshoe vortex has legs that rotate such as to induce upwards flow, whereas the inverted horseshoe vortex induces downward flow. The horseshoe head connects the two legs of an upright vortex at its upstream end and those of an inverted vortex at its downstream end. On a vertical/streamwise plane and with the flow moving from left to right, the heads of both upright and inverted vortices rotate clockwise, which is consistent with PIV measurements which showed a predominance of clockwise vortices in the vertical plane. The lengths and orientations of both upright and inverted horseshoes are comparable. The present configuration of an inverted horseshoe vortex coincides with the second characteristic vortex found by Rogers \& Moin (1987).

A possible mechanism that explains the presence of both upright and inverted horseshoe vortices in USF is illustrated in figure 16. Similar sketches have been presented by Smith et al. (1991) and Robinson (1991) to describe the development of a symmetric hairpin. Moreover, the shape of the vortex on the right side of figure 16 is consistent with the presence of forward and backward-facing arches apparent in the hydrogen bubbles timelines in figure 8b, as well as other evidence from dye visualisation and PIV maps. This mechanism may be briefly described as follows. Spanwise, 'roller-type' vortices may exist in USF as remnants of those produced by the shear generation apparatus or could 


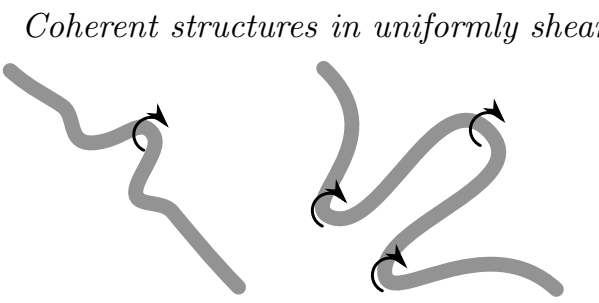

FIGURE 16. Conceptual sketch of a possible mechanism for the generation of horseshoe vortices in USF. A spanwise vortex created by some instability mechanism or present in the flow first deforms due a small disturbance. The deformed vortex is then inclined and stretched by the shear, resulting in both upright and inverted horseshoe vortices.

be generated by the interaction of some instability mechanism with the mean vorticity of the flow. Because USF is unhindered by the presence of a wall, the mean strain stretches and realigns these roller vortices in both upwards and downwards directions, at approximately $45^{\circ}$ and $-135^{\circ}$, thus resulting in both upright and inverted horseshoes. The same vortex stretching and realignment mechanism is also present in TBL, but it may be conjectured that obstruction by and friction with the wall act as to suppress the development of the inverted structures, leaving only the upright ones. Rogers \& Moin (1987) suggested that hairpin vortices are an important vortical structure in all turbulent shear flows, but did not elaborate on the effect that the wall may have on the orientation of such structures. As far as the present authors are aware, such an effect has not been described in previous literature.

\subsection{Vortex packets and zones of nearly uniform velocity}

A previous observation in TBL is that horseshoe/hairpin vortices travel in groups, referred to as packets (Adrian 2007). A similar observation was made in the present USF: the streamwise alignment of multiple heads appearing in PIV velocity maps in a vertical plane indicated the presence of groups of quasi-streamwise-aligned horseshoe vortices. Groups of three to six vortex heads were often observed travelling together, forming chains that stretched up to $100 \mathrm{~mm}$ in length.

In TBL, vortex packets create zones of uniform velocity through the effect of 'coherent vortex induction' inside the packets (Adrian 2007). In the current study of USF, instantaneous velocity maps also contained zones of roughly uniform velocity. Across the boundaries of these locally uniform zones, the instantaneous velocity profiles underwent relatively large step-like changes, in sharp contrast to the near-linearity of the time-averaged velocity profile. In the vertical plane, these zones were flanked by shear layers. In the horizontal plane, these zones were manifested as quasi-streamwise streaks of low- and high-speed fluid. In the inclined plane, these zones caused the velocity contours to form saw-tooth patterns in the spanwise direction. In all three measurement planes vortices were identified in the shear layers between these zones.

The zones of nearly uniform velocity are observed in both TBL and USF and are a sign characteristic of the presence of horseshoe vortices. The low-speed pockets are representative of the upward flow induced by packets of quasi-streamwise-aligned, upright horseshoe vortices. In USF, but not in TBL, packets of quasi-streamwise-aligned, inverted structures generated high-speed pockets of fluid. The currently observed zones of uniform velocity were about twice as tall as they were wide, in conformity with the elongated shape of the corresponding horseshoes. The fairly long streamwise length of the uniform-velocity zones is attributed to the streamwise alignment of two or more horseshoe structures. Figure 17 displays a low-speed zone of typical proportions with several fitted upright 


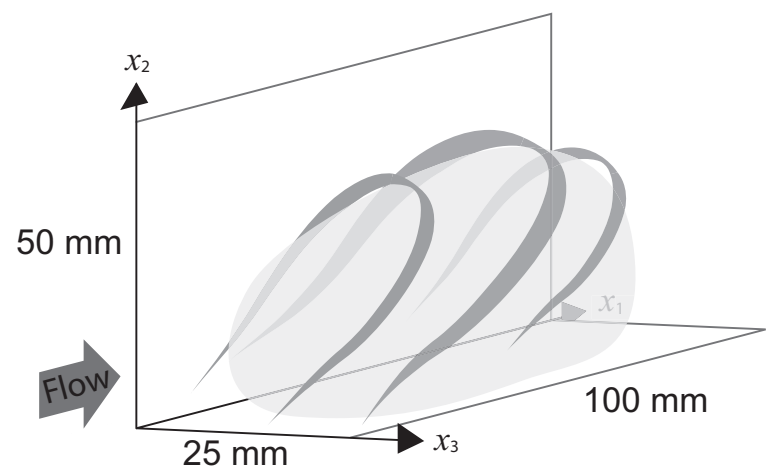

FIGURE 17. Conceptual illustration of a packet of upright horseshoe vortices that produces a low-speed zone of nearly uniform velocity in USF.

horseshoe vortices. This sketch is reminiscent of hairpin packets in TBL, as described by Adrian (2007).

\subsection{The formation of horseshoe vortices in USF}

It has been firmly established that, in homogeneous fluids, vorticity may only be generated at solid boundaries of the flow (Morton 1984). Considering that the effects of the side and bottom walls on the core region of the present USF are negligible, one may confidently assess that all vortices in USF contain vorticity generated by the shear generator/flow separator apparatus. In this section, we shall attempt to explain the physical process by which the highly organized, jet-wake-like vortex field at the exit of the sheargenerating apparatus evolves into the vastly different field of horseshoe-like coherent structures in fully-developed USF.

As illustrated previously, the flow at the exit of the shear-generating apparatus contained an abundance of strong clockwise and counter-clockwise vortices with roughly equal concentrations. The strengths of the two sets also appear not to be very different, however, the total vorticity magnitude of regions with clockwise rotation (including coherent and non-coherent ones) must be higher than that of regions with counter-clockwise rotation, as the difference between these two values is equal to the mean vorticity of USF. As the flow developed from $0.1<\tau<5$, the total number of vortices that could be identified decreased and the proportion of counter-clockwise vortices decreased as well. Beyond $\tau=5$, the clockwise vortices were clearly predominant and they were organized along strong shear layers, which did not align horizontally with the wakes of the shear-generating apparatus.

As the flow develops, the discrete vortices evolve through interactions with other vortices as well as under the influence of the mean flow vorticity. The merging of a clockwise vortex with a counter-clockwise one of comparable strength may cancel their vorticities, whereas the merging of two vortices with the same sense of rotation would add their strengths. Because the apparatus generates alternating vortices, it is likely that each vortex would interact preferentially with one of the opposite sense of rotation and so the total number of vortices decreases away from the apparatus. At the same time, the interaction between the mean vorticity of the flow and discrete vortices would tend to strengthen the clockwise vortices while cancelling partially or totally the vorticity of the counter-clockwise vortices, thus shifting the balance in favour of clockwise vortices. These processes are consistent with our observations using a traversing PIV system, which showed that, in general, the strong clockwise vortices persisted for the length of 
our measurement section, whereas the counter-clockwise vortices disappeared after some distance; our efforts to detect any spontaneous generation of new vortices in USF did not culminate in producing such evidence.

The previous discussion has so far outlined a plausible mechanism that explains the evolution of a jet-wake-like vortical field towards one dominated by spanwise vortices with predominantly clockwise rotation. The evolution of the latter into horseshoe vortices may be attributed to the mechanism described by Smith et al. (1991) and Davidson (2004) for TBL: in the presence of disturbances, spanwise vortex filaments would stretch and fold in the direction of mean strain, thus giving rise to horseshoe vortices. This mechanism redirects some spanwise vorticity to a quasi-streamwise one.

In summary, our experimental evidence and the associated analysis seem to indicate that the vorticity of each horseshoe vortex in USF can be traced back to the sheargenerating apparatus. Nevertheless, this by no means signifies that the structure of fullydeveloped USF is predetermined by its initial state and that it is apparatus-specific. Our results show in the clearest terms that the organization of the coherent structures far from the entrance is entirely different from the one near it. It is the presence of mean vorticity (mean shear) that establishes the distinct horseshoe vortex field of USF. Without sufficient mean shear, spanwise vortex rotation would not have a preferential orientation and horseshoe vortices would not align themselves preferentially in the observed manner. Although we do not have any direct supporting evidence, we may speculate that USF with different initial structures would eventually acquire coherent structures which may at most depend on the Reynolds number and/or the shear parameter, but not the initial condition.

The present postulates are supported by the similarity of coherent structures in USF and HSF (see §6.6). In HSF, which has initially uniform vorticity and no organized initial vortices, part of the mean vorticity is partitioned into predominantly clockwise spanwise vortices by means of a Kelvin-Helmholtz instability mechanism. Kiya \& Arie (1979) showed mathematically that a vortex sheet in the presence of uniform shear is unstable with respect to small disturbances, and that its roll-up process is promoted by background vorticity. Vincent \& Meneguzzi (1994), using DNS, observed that discrete vortices were created by the disintegration of vortex sheets even in unsheared homogeneous turbulence. The mean shear would then transform these discrete vortex filaments into horseshoe vortices, as in USF and TBL (Rogers \& Moin 1987). In low-disturbance HSF, the actions of this instability mechanism would be relatively slow, whereas in USF the presence of strong initial vortices would likely accelerate the process. It is relevant to note that Kim et al. (2008) showed that increasing background noise in TBL enhances the generation of horseshoe vortices.

\subsection{The effects of $R e_{\lambda_{11}}$ and $S^{*}$}

The turbulence in USF may be characterized by the values of the shear parameter $S^{*}$ and the turbulence Reynolds number $R e_{\lambda_{11}}$. As shown in table 4, the time-averaged turbulence properties of the current USF are not very different from those in previous realizations with comparable values of $R e_{\lambda_{11}}$ and $S^{*}$.

Previous authors have investigated the effect of $R e_{\lambda_{11}}$ and $S^{*}$ on the turbulence properties and in particular on the shear stress anisotropy $m_{12}$, which, in general, is the most sensitive indicator of the turbulence structure (De Souza et al. 1995). Based on DNS and windtunnel experiments, Isaza et al. (2009) found that the turbulence properties are most sensitive to $S^{*}$, and that $m_{12}$ tends to decrease with increasing $S^{*}$. De Souza et al. (1995) investigated USF with high shear in a high-speed windtunnel and found a similar decrease in $m_{12}$ compared to 'low-shear' USF. A numerical investigation by 


$\begin{array}{lcccc} & \text { present } & \text { TC } & \text { TK } & \text { IWC } \\ R_{\lambda_{11}} & 150 & 128 & 160 & 159 \\ S^{*} & 13 & 12.6 & 9 & 10 \\ a & 0.12 & \text { n.a. } & 0.1 & \text { n.a. } \\ \epsilon / P r & 0.6 & 0.55 & 0.69 & 0.66 \\ m_{12} & -0.15 & -0.14 & -0.16 & -0.12 \\ \max \tau & 11 & 12 & 28 & 16\end{array}$

TABLE 4. Comparison of the turbulence properties in the current USF to previous works with comparable $R e_{\lambda_{11}}$ and $S^{*}$ : (TC) Tavoularis \& Corrsin (1981), (TK) Tavoularis \& Karnik (1989), and (IWC) Isaza et al. (2009).

Shih et al. (2000), however, suggested that at higher values of $R e_{\lambda_{11}}$, the turbulence properties are more sensitive to $R e_{\lambda_{11}}$ than to $S^{*}$. Ferchichi \& Tavoularis (2000) also investigated experimentally the effect of $R e_{\lambda_{11}}$ and confirmed that $m_{12}$ decreases with increasing $R e_{\lambda_{11}}$.

In terms of coherent structures, Head \& Bandyopadhyay (1981) explained that an increase in the Reynolds number tended to elongate the horse-shoe vortices into hairpin shapes. In HSF, Lee et al. (1990) described a similar elongation of the flow structures, and attributed it to an increase in the shear parameter.

In summary, there seems to be sufficient evidence that, as $R e_{\lambda_{11}}$ or $S^{*}$ increase, $m_{12}$ decreases and the coherent structures become more elongated. De Souza et al. (1995) suggested that this trend is typical of both USF and TBL.

\subsection{USF vs. HSF and TBL}

As discussed previously, the large-scale structure of USF has a strong resemblance to those of HSF and the outer region of TBL. In this section, we shall emphasize on the qualitative differences among these flows and attempt a quantitative comparison. All vorticity in both USF and HSF is present at the time of their generation, whereas vorticity is continuously generated along TBL. Discrete vortices are present at inception only in USF, whereas mechanisms generating discrete vortices from the mean vorticity exist in HSF and TBL. Horseshoe/hairpin vortices are encountered commonly in all three flows, but USF and HSF have both upright and inverted vortices, whereas TBL have only upright ones. Organized groups (packets) of horseshoe vortices have been observed in USF and TBL; although the existence of vortex groups in HSF has not been explicitly documented in the available literature, it seems plausible that such groups would emerge if the computational domain were sufficiently large. Smith et al. (1991) and Zhou et al. (1999) conclude that hairpin vortices in TBL near the wall are capable of generating others that together form the vortex packet. In USF, we have not observed the generation of new vortices, and so we conjecture that packets are formed by the organization of preexisting discrete vortices.

In order to compare the characteristic dimensions of coherent structures in the three flows of interest, we require the use of an appropriate length scale. Among the available scales, the most relevant one appears to be the Taylor microscale $\lambda_{11}$. According to figure 1 in the note by Tennekes (1968), this scale represents the distance between branches of looping vortex tubes. As presented in table 5, the distances between the legs of horseshoe vortices in the present USF, as well as in HSF (Rogers \& Moin 1987) and in TBL (Adrian et al. 2000) with not too distant values of turbulent Reynolds number, are comparable to the streamwise Taylor microscale $\lambda_{11}$, in agreement with Tennekes' 


\begin{tabular}{lccc}
\hline & USF & HSF & TBL \\
$R_{\lambda_{11}}$ & 150 & 142 & 189 \\
$S^{*}$ & 13 & $9-12$ & n.a. \\
Length of structures & $3.2 \lambda_{11}$ & $3.2 \lambda_{11}$ & $2 \lambda_{11}\left(\approx 200 \delta_{\nu} \approx 0.24 \delta\right)$ \\
Width of structures & $1.6 \lambda_{11}$ & $1.6 \lambda_{11}$ & $\lambda_{11}\left(\approx 100 \delta_{\nu} \approx 0.12 \delta\right)$ \\
Diameter of vortices & $0.3 \lambda_{11}$ & n.a. & $0.3 \lambda_{11}\left(\approx 30 \delta_{\nu} \approx 0.04 \delta\right)$ \\
Inclination of structures & $30^{\circ}-45^{\circ}$ & $35^{\circ}-40^{\circ}$ & $25^{\circ}-45^{\circ}$ \\
Number of structures in a packet & $3-5$ & n.a. & $3-10$ \\
Height of packets & $3 \lambda_{11}$ & n.a. & $7 \lambda_{11}\left(\approx 700 \delta_{\nu} \approx 0.8 \delta\right)$ \\
Length of packets & $6 \lambda_{11}$ & n.a. & $17 \lambda_{11}\left(\approx 1700 \delta_{\nu} \approx 2 \delta\right)$
\end{tabular}

TABLE 5. Comparison of the properties of fully developed hairpin structures in the current USF, in HSF (Rogers \& Moin 1987), and in a TBL (Adrian et al. 2000).

postulate. The lengths of these structures in all three flows are two to three times $\lambda_{11}$. The normalized diameters of vortices in USF and TBL are about the same and their inclinations have comparable ranges in the three flows. Moreover, table 5 shows that the typical number of structures in a packet in TBL is measurably larger than that in USF; the heights and lengths of the packets in TBL are also larger than the corresponding dimensions in USF. It is noted that most observations in TBL are reported in terms of either the boundary layer thickness $\delta$ or the viscous length scale $\delta_{\nu}=\nu / \sqrt{\tau_{w} / \rho}$, where $\tau_{w}$ is the wall shear stress; to permit further comparisons, scaling of horseshoe vortices in the TBL in terms of these two parameters has also been included in table 5. Because Rogers \& Moin (1987) did not explicitly report the average length of the structures they observed, we estimated the ratio of the length to the width of the structures as 2:1 based on the example structures they presented. The values reported in the table are likely to change as the Reynolds number of any of these three flows increases significantly; as discussed previously, the ratio of the length and width of the structures is expected to increase with increasing Reynolds number.

\section{Conclusions}

Uniformly sheared flow was successfully reproduced in a water tunnel, with characteristics comparable to those in previously published experiments. A turbulence Reynolds number $R_{\lambda_{11}}$ of approximately 150 was obtained, as well as a shear coefficient $S^{*}$ of approximately 13. Laser Doppler velocimetry was used to verify that the turbulent properties agreed with conventional values.

In this research, we have been the first to document experimentally and quantitatively the presence of horseshoe-shaped vortices in USF. Horseshoe-shaped structures were observed using flow visualisation and PIV. Scanning dye visualisation identified the presence of elongated quasi-streamwise vortex pairs, which were interpreted as the legs of horseshoe vortices. Vortex pairs were observed having a range of orientations, which included both upright and inverted horseshoe structures. Using hydrogen bubble visualisations, the heads of the horseshoe structures were observed to connect quasi-streamwise legs. The properties of these vortices were also measured quantitatively using PIV and were in general agreement the observations from the flow visualisations. Furthermore, the instantaneous flow was observed to consist of zones of roughly uniform velocity separated by strong shear layers, rather than having uniform shear. These zones of roughly uniform velocity had a similar average spanwise width to that of the individual horseshoe struc- 
tures and were understood to be formed by the quasi-streamwise alignment of several horseshoe vortices.

The coherent, horseshoe/hairpin-like vortices observed in USF appear to be similar to those observed by previous authors in HSF and in TBL. These results further support the hypothesis that such vortices are generated by mean shear, irrespectively of the presence of a solid wall. Furthermore, our observations suggest that the dominant coherent structures of fully developed USF are very different from the structures observed in the flow exiting the shear-generating apparatus, which points to an insensitivity of the former to initial effects.

Financial support by Le Fonds Québécois de la Recherche and by the Natural Sciences and Engineering Research Council of Canada (NSERC) is gratefully acknowledged.

\section{Appendix A. Details of the facility and procedures}

\section{A.1. LDV system}

A two-component, fibre-optic, laser Doppler velocimeter with a burst analyzer (Model BSA F50 60N20, Dantec Dynamics A/S, Skovlunde, Denmark), operating in backscatter mode and powered by a $5 \mathrm{~W}$ Argon-ion laser (Model 95L-5, Lexel Laser, Fremont, California, USA), was used for measuring the time-averaged statistics of the flow. For most measurements, the LDV probe was mounted beside the test section with its axis aligned with the $x_{3}$-direction, allowing for measurements of the streamwise $U_{1}$ and vertical $U_{2}$ velocity components; for measurements of $U_{3}$, the probe was mounted below the test section with its axis aligned with the $x_{2}$-direction. A lens with a focal distance of $310 \mathrm{~mm}$ and a beam expander (Dantec Dynamics Model 55X12) were used for these measurements. The measuring volume was approximately ellipsoidal with a length of $865 \mu \mathrm{m}$ and a height/width of $77 \mu \mathrm{m}$. Silicon carbide micro-spheres with a diameter of $2 \mu \mathrm{m}$ (TSI, Shoreview, Minnesota, USA) and a time constant of about $0.82 \mu$ s were used as seeds.

\section{A.2. Hydrogen bubble visualisation procedure}

Hydrogen bubbles were created in the flow by the electrolysis of water achieved by applying an electric voltage of approximately $300 \mathrm{~V}$ between two immersed electrodes. The bubbles were released from the cathode, which was a platinum wire with a diameter of $50 \mu \mathrm{m}$ and a length of $380 \mathrm{~mm}$. Oxygen bubbles were also released from a carbon rod serving as the anode but placed away from view. Sodium sulphate was added to the water to increase its electrical conductivity. A home-made electronic circuit was used to generate electric pulses with the desired frequency and duty cycle. The bubble diameter was estimated to be about $25 \mu \mathrm{m}$ and the bubble terminal rise velocity was estimated to be about $0.3 \mathrm{~mm} / \mathrm{s}$, which was sufficiently low for buoyancy-related errors to be insignificant in the present experiments. Still images of the bubbles were recorded using a Nikon D70 Digital SLR camera with a lens of adjustable focal length in the range of 18-70 $\mathrm{mm}$. The images were digitally enhanced using Adobe Photoshop to increase the brightness and contrast of the bubbles with respect to the background.

\section{A.3. Dye visualisation procedure}

Planar flow visualization was made possible by laser-sheet illumination of injected fluorescent dyes that were premixed with water. Two fluorescent dyes, dibromofluorescein and fluoroscein sodium salt, were used. Because the molecular diffusion of these dyes is negligible, they maintained excellent visibility throughout the test section, even though 
they were injected upstream of the shear generator to avoid flow disturbance by the injection tube. The light sheet was generated from the previously mentioned Argon-ion laser, whose output was split into two parts; one part was directed into the LDV system, whereas the other part was led into a fibre-optic cable connected to a Powell lens $(\mathrm{Oz}$ Optics, Ottawa, Canada), which created a light sheet with an approximate thickness of $2 \mathrm{~mm}$. The laser light had spectral peaks at two wavelengths, $488.0 \mathrm{~nm}$ and $514.5 \mathrm{~nm}$, and provided sufficient excitation to the fluorescent molecules, which had an absorption peak at approximately $495 \mathrm{~nm}$ and an emission peak at approximately $517 \mathrm{~nm}$.

Volumetric displays of the dye patterns in the flow were reconstructed by analysis of images obtained using a specially designed flow scanner, illustrated in figure 18. This consisted of a carriage riding along rails positioned under the test section and on which both the camera and the laser-sheet lens were mounted rigidly. The carriage was set in motion towards the upstream by the action of a gate latch, which released a falling weight of approximately $93 \mathrm{~N}$, tethered to the carriage through a steel cable and a pulley. As the carriage approached the upstream end of the test section, it caused a brake to make contact with an inclined surface located beside the rails, which gradually increased friction and brought the carriage to a stop without significant jolt. The instantaneous position of the carriage during the scan was recorded by the camera simultaneously with the flow patterns by recording the image of a ruler positioned under the carriage and reflected in a mirror. Flow images were recorded with the use of a high-speed camera (Fastcam 512 PCI, Photron Inc., San Diego, California, USA) at a rate of 500 frames/s . Each recorded image captured a flow area of approximately $250 \mathrm{~mm} \times 250 \mathrm{~mm}$. The cart position and velocity from one representative scan are plotted in figure 19. Following an acceleration time of $0.4 \mathrm{~s}$, the carriage surpassed a speed of $1 \mathrm{~m} / \mathrm{s}$, reaching a maximum speed of $1.6 \mathrm{~m} / \mathrm{s}$. The carriage reached the end of its travel after approximately $0.9 \mathrm{~s}$ of travel. Images taken before the cart reached $1 \mathrm{~m} / \mathrm{s}$ and after the brake was applied were discarded. With a frame rate of 500 frames/s, the streamwise spacing of the images was approximately $3 \mathrm{~mm}$ while the carriage was travelling at its maximum velocity. Considering that the centreline velocity of the flow was about $0.16 \mathrm{~m} / \mathrm{s}$ travelling in the opposite direction, the flow would have travelled by approximately $80 \mathrm{~mm}$ during the last $0.5 \mathrm{~s}$ of the scan. This caused streamwise measurements of the flow domain to be foreshortened by approximately $10 \%$, but this error was corrected for in the presented results. Because vertical and spanwise flow velocities were much smaller, any flow movement in these directions during the scan is considered to be negligible.

The image sequence recorded during the scan was converted into a volumetric display by resampling the image data into an orthogonal three-dimensional array of voxels filling the entire measurement volume. Because the carriage speed was not uniform, the spacing of the image planes was also non-uniform; the actual image spacing was determined from the recorded images of the ruler in each frame. The voxel array was exported from Matlab and analyzed using ITK-SNAP (www.itksnap.org) to perform the image segmentation.

\section{A.4. PIV system and procedures}

The PIV system comprised a Nd:YAG, dual-cavity, pulsed laser (Solo PIV 120XT, New Wave Research, Inc., Fremont, California, USA), which provided up to $120 \mathrm{~mJ}$ of power per pulse at a frequency-doubled wavelength of $532 \mathrm{~nm}$. Light sheet optics formed a laser sheet with a thickness of approximately $2 \mathrm{~mm}$. A digital video camera (Imager ProX 4M, LaVision Inc., Ypsilanti, Michigan, USA) with a resolution of $2048 \times 2048$ pixels recorded image pairs at a maximum recording rate of 7.24 frames/s, achieved with the use of interframe recording. The camera was fitted with a Nikon AF Nikkor $50 \mathrm{~mm} f / 1.8 \mathrm{D}$ lens with an adjustable focal length in the range of $18-70 \mathrm{~mm}$ and a band-pass interference 


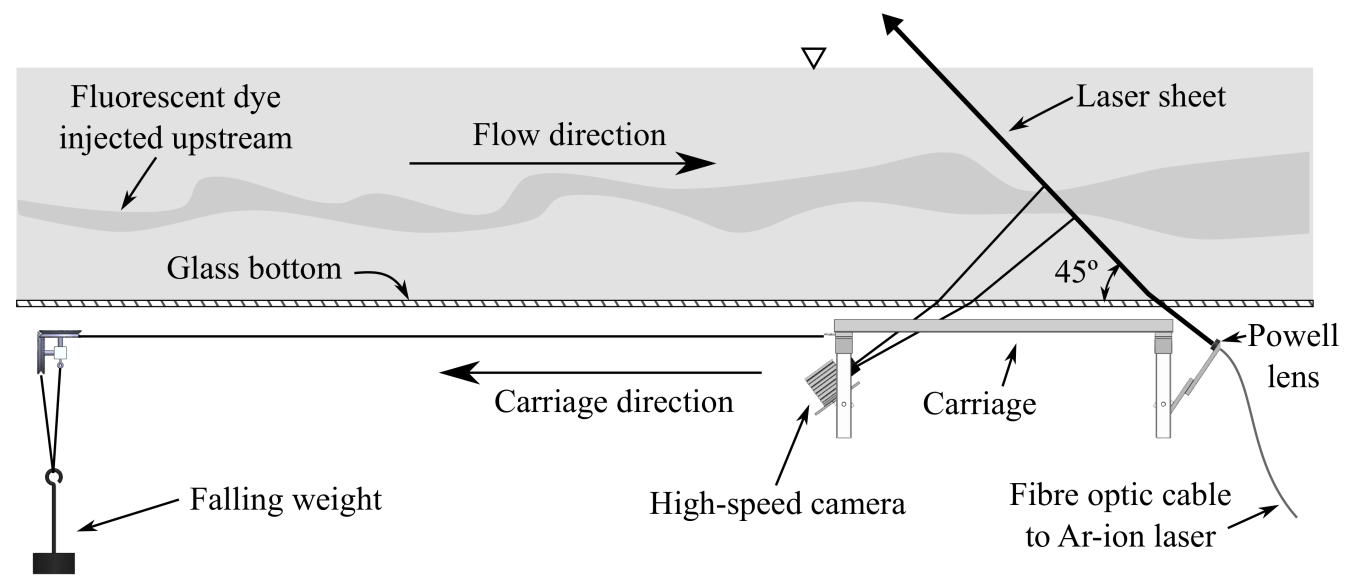

FigURE 18. Illustration of the carriage system used to scan a laser sheet through the test section in order to capture volumetric dye patterns.
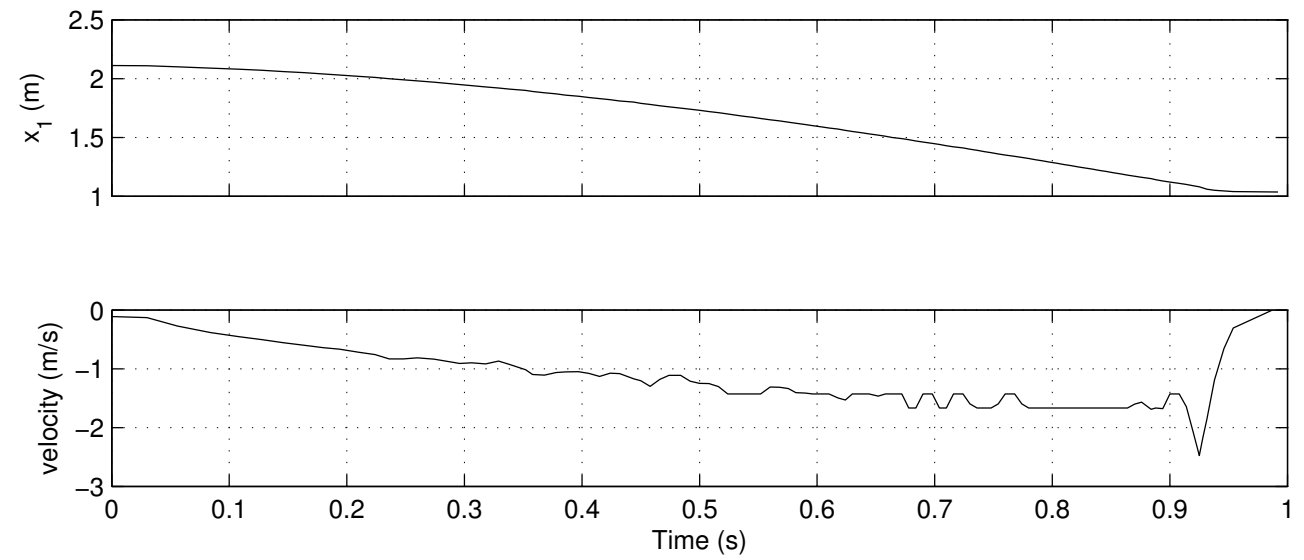

FiguRE 19. Measurement of the carriage position and velocity. The cart is released at $\mathrm{t}=0 \mathrm{~s}$ and the brake is actuated at $\mathrm{t}=0.9 \mathrm{~s}$. Images were recorded at a rate of 500 frames per second and those occurring before the carriage reached a speed of $1 \mathrm{~m} / \mathrm{s}$ or after the brake was actuated were discarded.

filter (LaVision Model 1108501) that only allowed light in a narrow waveband centred at $532 \mathrm{~nm}$. A programmable timing unit (LaVision Model 1108058) synchronized the camera and the laser operations. Velocity vector field extraction from the digital images was performed entirely by software provided by the manufacturer (Davis software, LaVision). The final result was a vector field of $128 \times 128$ vectors for the entire field of view that was set at $100 \mathrm{~mm} \times 100 \mathrm{~mm}$. Thus the spatial resolution of the PIV map was approximately $0.8 \mathrm{~mm}$. Flow seeding was the same as that used for the LDV measurements.

PIV measurements were taken at three different planar orientations: (a) a vertical orientation containing the flow direction and for which the camera was mounted on the side traversing system and the laser was mounted on the bottom traversing system; (b) a horizontal orientation for which the laser was positioned beside the channel and the camera was mounted on a tripod underneath the channel; and (c) an inclined orientation, 
forming an angle of $45^{\circ}$ with the flow direction and such that the upper part of the viewing plane was towards the upstream. For the latter configuration, the light sheet was directed into the test section by a tilted mirror, mounted together with the laser on the bottom traversing system, and the camera was mounted tilted upwards on the tripod underneath the channel.

The areas of the vortex cores were determined by using an area growing algorithm starting at each peak of swirling strength. This algorithm identified the extent of the vortex core as the coincident region with a swirling strength greater than $0.025 \lambda_{\max }$. In most cases, this approach made it possible to separate discrete vortices, especially those of opposite vorticity, as the swirling strength vanishes when the vorticity changes sign. However, in some cases, a single vortex may have had multiple peaks in swirling strength, which happened when the swirling strength had a local minimum that was higher than $0.025 \lambda_{\max }$; multiple peaks may have indicated vortices in the process of splitting or merging. The size of each vortex was calculated as the length of the major axis of the minimum-area ellipse that enclosed the vortex.

The preliminary set of vortices identified in each of the time frames was then screened for cross-frame consistency in order to remove noise-produced peak detections and weak vortices. All vortices that were identified but could not be followed in one of the two adjacent time frames were discarded. During this screening, the change in position of the vortex based on its convection speed was accounted for and changes in vortex size and strength by up to $50 \%$ were tolerated. This screening successfully removed most of the small vortices that were indistinguishable from noise and ensured that the reconstructed coherent structures had some temporal continuity.

\section{Appendix B. Further analysis of the horizontal PIV measurements}

The three-dimensional orientation of each identified vortex can be estimated by assuming that each ellipse that was fitted to a high-swirl region in a horizontal PIV map was the cross-section of a cylindrical vortex tube. Then, the orientation of the projection of the vortex tube axis on the horizontal plane can be determined from the angle $\alpha$ between the major axis of the ellipse and the streamwise direction. Furthermore, the angle of inclination $\beta$ of the vortex tube with respect to the horizontal plane can be calculated from the ellipticity (i.e., the ratio of the two axes of the ellipse) as

$$
\beta=\sin ^{-1}\left(\frac{L_{\text {major axis }}}{L_{\text {minor axis }}}\right) \text {. }
$$

The joint p.d.f. of the two angles $\alpha$ and $\beta$ is presented in figure 20. The highest peak of this j.p.d.f. indicates that vortex tubes passing through the horizontal plane are most often inclined by $\beta \approx 35^{\circ}$ in the streamwise direction $\left(\alpha=0^{\circ}\right)$. Although in determining the angle $\beta$ we were unable to distinguish whether the vortex tube was facing upstream or downstream, it is expected that the vast majority of structures would be leaning forward, in conformity with the direction of the shear and with the observations from the hydrogen bubbles and dye visualisations. As expected, the j.p.d.f. is approximately symmetric about the streamwise axis $\left(\alpha=0^{\circ}\right)$, in conformity with the spanwise homogeneity of USF. Two lower peaks may be observed at $\alpha \approx \pm 50^{\circ}$ and $\beta \approx 40^{\circ}$. A peak at a nonzero $\alpha$ implies that there is another preferential inclination of vortex tubes other than a streamwise one. This could occur during the evolution of spanwise rollers into horseshoe vortices, at which time the legs of the horseshoe may be divergent.

In order to test the hypothesis that at least a large percentage of the identified vortices are legs of horseshoe-shaped vortices, it is helpful to examine statistically whether 


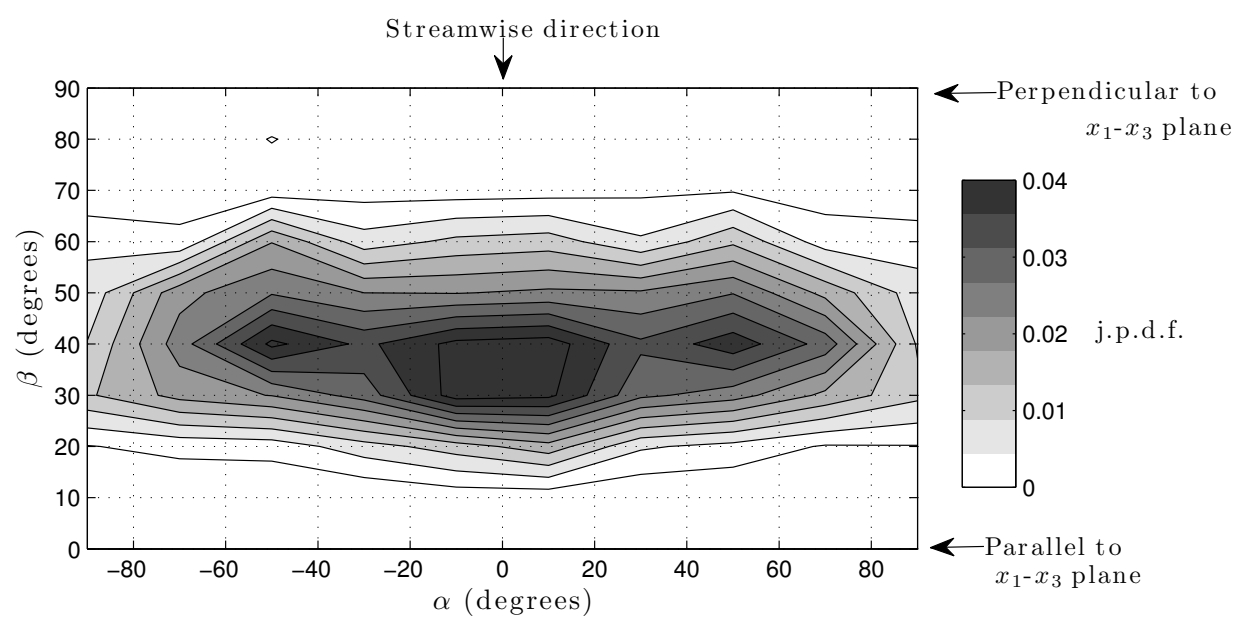

FigurE 20. The j.p.d.f. of the angles $\alpha$ between the major axes of the ellipses that denote vortices in the horizontal plane and the streamwise direction and the angles of inclination $\beta$ of the vortex tubes with respect to the horizontal plane.

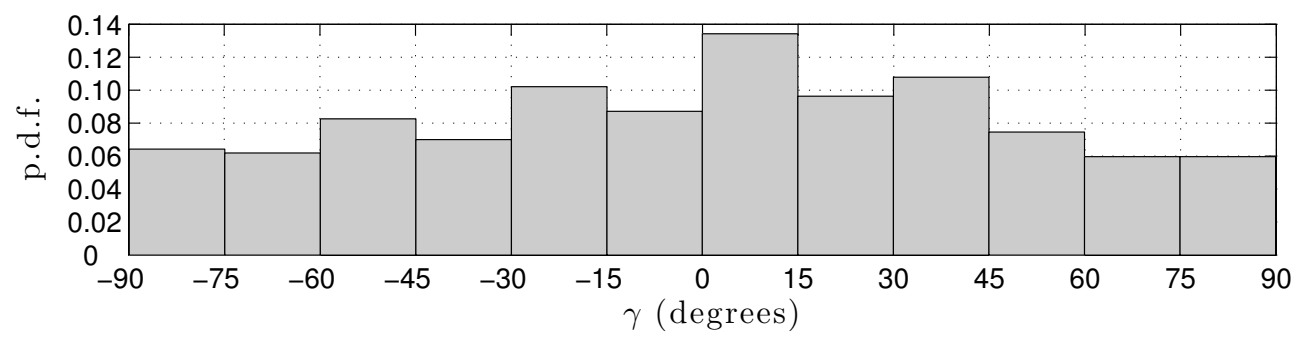

FiguRE 21. The p.d.f. of the angle $\gamma$ of the direction normal to the line connecting the two vortices of each pair identified with PIV in the horizontal plane, with respect to the streamwise direction. The inclinations were determined by the angle of the line connecting the centres of the minimum-area ellipses that enclosed each vortex.

each clockwise vortex can be paired with a counter-clockwise counterpart and whether the pair bestrides a low- or high-speed region. Towards this purpose, a pair-matching algorithm was developed in order to help determine vortex pairs free of subjective bias. This algorithm identified pairs based on closeness in strength, convection velocity, and physical proximity between each clockwise vortex and each counter-clockwise vortex. Representative results are presented in figure 13, in which the vortex pairs are connected by solid black lines. In the horizontal plane, the algorithm matched an average of nine pairs per frame. The p.d.f. of the angle $\gamma$ between the streamwise direction and the direction normal to the line connecting the two vortices of each pair is presented in figure 21. This figure illustrates that vortex pairs, and consequently horseshoe vortices, are more likely to travel with their plane facing the flow direction rather than sideways.

\section{REFERENCES}

Adrian, R. J. 2007 Hairpin vortex organization in wall turbulence. Phys. Fluids 19, 041301.

Adrian, R. J., Meinhart, C. D. \& Tomkins, C. D. 2000 Vortex organization in the outer region of the turbulent boundary layer. J. Fluid Mech. 422, 1-54. 
Adrian, R. J. \& Moin, P. 1988 Stochastic estimation of organised turbulent structure: homogeneous shear flow. J. Fluid Mech. 190, 531-559.

Davidson, P. A. 2004 Turbulence - An Introduction for Scientists and Engineers. Oxford University Press.

De Souza, F. A., Nguyen, V. D. \& Tavoularis, S. 1995 The structure of highly sheared turbulence. J. Fluid Mech. 303, 155-167.

FALCO, R. E. 1977 Coherent motions in outer region of turbulent boundary layers. Phys. Fluids 20, S124-S132.

Ferchichi, M. \& TAvoularis, S. 2000 Reynolds number effects on the fine structure of uniformly sheared turbulence. Phys. Fluids 12, 2942-2953.

Gao, Q., Ortiz-Duenas, C. \& Longmire, E. K. 2007 Circulation signature of vortical structures in turbulent boundary layers. In 16th Australasian Fluid Mechanics Conference (ed. P. Jacobs, T. McIntyre, M. Cleary, D. Buttsworth, D. Mee, R. Clements, R. Morgan \& C. Lemckert), pp. 135-141. School of Engineering, The University of Queensland, Brisbane, Australia.

Head, M. R. \& Bandyopadhyay, P. 1981 New aspects of turbulent boundary-layer structure. J. Fluid Mech. 107, 297-338.

IsazA, J. C. \& Collins, L. R. 2009 On the asymptotic behaviour of large-scale turbulence in homogeneous shear flow. J. Fluid Mech. 637, 213-239.

Isaza, J. C., Warhaft, Z. \& Collins, L. R. 2009 Experimental investigation of the large-scale velocity statistics in homogeneous turbulent shear flow. Phys. Fluids 21, 065105.

KIDA, S. \& TANAKA, M. 1994 Dynamics of vortical structures in a homogeneous shear flow. $J$. Fluid Mech. 274, 43-68.

Kim, K., Sung, H. J. \& Adrian, R. J. 2008 Effects of background noise on generating coherent packets of hairpin vortices. Phys. Fluids 20, 105107.

Kislich-Lemyre, B. 2002 The large-scale structure of uniformly sheared turbulence and its distortion by a solid wall at rest or in motion. PhD Dissertation, Department of Mechanical Engineering, University of Ottawa, Ottawa, Canada.

KIYA, M. \& ARIE, M. 1979 Helmholtz instability of a vortex sheet in uniform shear flow. Phys. Fluids 22, 378-379.

Kline, S. J., Reynolds, W. C., Schraub, F. A. \& Runstadler, P. W. 1967 The structure of turbulent boundary layers. J. Fluid Mech. 30, 741-773.

Lee, M. J., Kim, J. \& Moin, P. 1990 Structure of turbulence at high shear rate. J. Fluid Mech. 216, 561-583.

Morton, B. R. 1984 The generation and decay of vorticity. Geophys. \& Astrophys. Fluid Dyn. 28, 277-308.

Pope, S. 2000 Turbulent Flows. Cambridge University Press.

Robinson, S. K. 1991 Coherent motions in the turbulent boundary layer. Annu. Rev. Fluid Mech. 23, 601-639.

Rogallo, R. S. 1981 Numerical experiments in homogeneous turbulence. NASA STI/Recon Technical Report N 81, 31508.

Rogers, M. M. \& Moin, P. 1987 The structure of the vorticity field in homogeneous turbulent flows. J. Fluid Mech. 176, 33-66.

Rohr, J. J., Itsweire, E. C., Helland, K. N. \& VAn Atta, C. W. 1988 An investigation of the growth of turbulence in a uniform-mean-shear flow. J. Fluid Mech. 187, 1-33.

Shen, X. \& WARHAFt, Z. 2000 The anisotropy of the small scale structure in high Reynolds number $\left(R_{\lambda} \sim 1000\right)$ turbulent shear flow. Phys. Fluids 12, 2976-2989.

Shin, L. H., Koseff, J. R., Ferziger, J. H. \& Rehmann, C. R. 2000 Scaling and parameterization of stratified homogeneous turbulent shear flow. J. Fluid Mech. 412, 1-20.

Smith, C. R., Walker, J. D. A., Haidari, A. H. \& Sobrun, U. 1991 On the dynamics of near-wall turbulence. Phil. Trans.: Phys. Sci. Eng. 336, 131-175.

TAvoularis, S. 1985 Asymptotic laws for transversely homogeneous turbulent shear flows. Phys. Fluids 28, 999-1001.

Tavoularis, S. 2005 Measurement in Fluid Mechanics. Cambridge University Press.

TAVoularis, S. \& Corrsin, S. 1981 Experiments in nearly homogeneous turbulent shear flow with a uniform mean temperature gradient. Part 1. J. Fluid Mech. 104, 311-347. 
TAVOULARIS, S. \& KARNIK, U. 1989 Further experiments on the evolution of turbulent stresses and scales in uniformly sheared turbulence. J. Fluid Mech. 204, 457-478.

Tennekes, H. 1968 Simple model for the small-scale structure of turbulence. Phys. Fluids 11, 669-671.

Vincent, A. \& Meneguzzi, M. 1994 The dynamics of vorticity tubes in homogeneous turbulence. J. Fluid Mech. 258, 245-254.

Wu, X. \& Moin, P. 2009 Direct numerical simulation of turbulence in a nominally zeropressure-gradient flat-plate boundary layer. J. Fluid Mech. 630, 5-41.

Zhou, J., Adrian, R. J., Balachandar, S. \& Kendall, T. M. 1999 Mechanisms for generating coherent packets of hairpin vortices in channel flow. J. Fluid Mech. 387, 353-396. 